ALEA, Lat. Am. J. Probab. Math. Stat. 18, 1241-1265 (2021)

DOI: $10.30757 /$ ALEA.v18-46

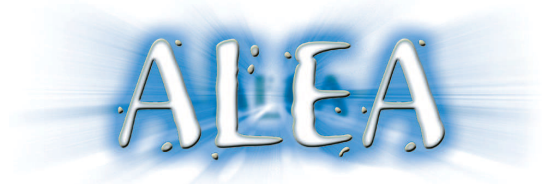

\title{
Convoluted Fractional Poisson Process
}

\author{
Kuldeep Kumar Kataria and Mostafizar Khandakar \\ Department of Mathematics \\ Indian Institute of Technology Bhilai \\ Raipur-492015, India. \\ E-mail address: kuldeepk@iitbhilai.ac.in, mostafizark@iitbhilai.ac.in
}

\begin{abstract}
In this paper, we introduce and study a convoluted version of the time fractional Poisson process by taking the discrete convolution with respect to space variable in the system of fractional differential equations that governs its state probabilities. We call the introduced process as the convoluted fractional Poisson process (CFPP). It has integer valued jumps of size $j \geq 1$. The explicit expression for the Laplace transform of its state probabilities are obtained whose inversion yields its one-dimensional distribution. A special case of the CFPP, namely, the convoluted Poisson process (CPP) is studied and its time-changed subordination relationships with CFPP are discussed. It is shown that the CPP is a Lévy process using which the long-range dependence property of CFPP is established. Moreover, we show that the increments of CFPP exhibits short-range dependence property.
\end{abstract}

\section{Introduction}

The Poisson process is a renewal process with exponentially distributed waiting times. This Lévy process is often used to model the counting phenomenon. Empirically, it is observed that the process with heavy-tailed distributed waiting times offers a better model than the ones with light-tailed distributed waiting times. For this purpose several fractional generalizations of the homogeneous Poisson process are introduced and studied by researchers in the past two decades. These generalizations give rise to some interesting connections between the theory of stochastic subordination, fractional calculus and renewal processes. These fractional processes can be broadly categorized into two types: the time fractional types and the space fractional types.

The time fractional versions of the Poisson process are obtained by replacing the time derivative in the governing difference-differential equations of the state probabilities of Poisson process by certain fractional derivatives. These include Riemann-Liouville fractional derivative (see Laskin, 2003), Caputo fractional derivative (see Beghin and Orsingher, 2009), Prabhakar derivative (see Polito and Scalas, 2016), Saigo fractional derivative (see Kataria and Vellaisamy, 2017b) etc. These time fractional models is further generalized to state-dependent fractional Poisson processes (see Garra et al., 2015) and the mixed fractional Poisson process (see Beghin, 2012 and Aletti et al., 2018). The

Received by the editors July 21th, 2020; accepted April 29th, 2021.

2010 Mathematics Subject Classification. 60G22, 60G55.

Key words and phrases. Time fractional Poisson process, discrete convolution, subordination, long-range dependence property, short-range dependence property. 
governing difference-differential equations of state-dependent fractional Poisson processes depend on the number of events that have occurred till any given time $t$. The properties related to the notion of long memory such as the long-range dependence (LRD) property and the short-range dependence (SRD) property are obtained for such fractional processes by Biard and Saussereau (2014), Maheshwari and Vellaisamy (2016), Kataria and Khandakar (2020).

Orsingher and Polito (2012) introduced a space fractional version of the Poisson process, namely, the space fractional Poisson process (SFPP). It is characterized as a stochastic process obtained by time-changing the Poisson process by an independent stable subordinator. Orsingher and Toaldo (2015) studied a class of time-changed Poisson processes associated with Bernštein functions. A particular choice of Bernštein function leads to a specific point process. Besides the SFPP, this class includes the relativistic Poisson process and the gamma-Poisson process as particular cases. Beghin and Vellaisamy (2018) introduced and study a process by time-changing the SFPP by a gamma subordinator. The jumps can take any positive value is a specific characteristics of these time-changed processes.

The time fractional Poisson process (TFPP), denoted by $\left\{N^{\alpha}(t)\right\}_{t \geq 0}, 0<\alpha \leq 1$, is a time fractional version of the homogeneous Poisson process whose state probabilities $p^{\alpha}(n, t)=\operatorname{Pr}\left\{N^{\alpha}(t)=\right.$ $n\}$ satisfy (see Laskin, 2003, Beghin and Orsingher, 2009)

$$
\partial_{t}^{\alpha} p^{\alpha}(n, t)=-\lambda p^{\alpha}(n, t)+\lambda p^{\alpha}(n-1, t), \quad n \geq 0,
$$

with $p^{\alpha}(-1, t)=0, t \geq 0$ and the initial conditions $p^{\alpha}(0,0)=1$ and $p^{\alpha}(n, 0)=0, n \geq 1$. Here, $\lambda>0$ is the intensity parameter.

The derivative $\partial_{t}^{\alpha}$ involved in (1.1) is the Caputo fractional derivative defined as

$$
\partial_{t}^{\alpha} f(t):=\left\{\begin{array}{l}
\frac{1}{\Gamma(1-\alpha)} \int_{0}^{t}(t-s)^{-\alpha} f^{\prime}(s) \mathrm{d} s, \quad 0<\alpha<1, \\
f^{\prime}(t), \quad \alpha=1
\end{array}\right.
$$

whose Laplace transform is given by (see Kilbas et al., 2006, Eq. (5.3.3))

$$
\mathcal{L}\left(\partial_{t}^{\alpha} f(t) ; s\right)=s^{\alpha} \tilde{f}(s)-s^{\alpha-1} f(0), \quad s>0 .
$$

For $\alpha=1$, the TFPP reduces to Poisson process.

The TFPP is characterized as a time-changed Poisson process $\{N(t)\}_{t \geq 0}$ by an independent inverse $\alpha$-stable subordinator $\left\{H^{\alpha}(t)\right\}_{t \geq 0}$ (see Meerschaert et al., 2011), that is,

$$
N^{\alpha}(t) \stackrel{d}{=} N\left(H^{\alpha}(t)\right)
$$

where $\stackrel{d}{=}$ means equal in distribution.

In this paper, we introduce a convoluted version of the TFPP by varying intensity as a function of states and by taking discrete convolution in (1.1). The discrete convolution used in (1.3) is defined in (2.1). We call the introduced process as the convoluted fractional Poisson process (CFPP) and denote it by $\left\{\mathcal{N}_{c}^{\alpha}(t)\right\}_{t \geq 0}, 0<\alpha \leq 1$. It is defined as the stochastic process whose state probabilities $p_{c}^{\alpha}(n, t)=\operatorname{Pr}\left\{\mathcal{N}_{c}^{\alpha}(t)=n\right\}$, satisfy

$$
\partial_{t}^{\alpha} p_{c}^{\alpha}(n, t)=-\lambda_{n} * p_{c}^{\alpha}(n, t)+\lambda_{n-1} * p_{c}^{\alpha}(n-1, t), \quad n \geq 0,
$$

with initial conditions

$$
p_{c}^{\alpha}(n, 0)= \begin{cases}1, & n=0 \\ 0, & n \geq 1\end{cases}
$$

and $p_{c}^{\alpha}(-n, t)=0$ for all $n \geq 1, t \geq 0$. Here, $\left\{\lambda_{j}, j \in \mathbb{Z}\right\}$ is a sequence of intensity parameters such that $\lambda_{j}=0$ for all $j<0$ and $\lambda_{j}>\lambda_{j+1}>0$ for all $j \geq 0$ with $\lim _{j \rightarrow \infty} \lambda_{j+1} / \lambda_{j}<1$. 
The Laplace transform of the state probabilities of CFPP is inverted to obtain its one-dimensional distribution in terms of Mittag-Leffler function, defined in (2.6), as

$$
p_{c}^{\alpha}(n, t)=\left\{\begin{array}{l}
E_{\alpha, 1}\left(-\lambda_{0} t^{\alpha}\right), \quad n=0, \\
\sum_{k=1}^{n} \sum_{\Theta_{n}^{k}} k ! \prod_{j=1}^{n} \frac{\left(\lambda_{j-1}-\lambda_{j}\right)^{k_{j}}}{k_{j} !} t^{k \alpha} E_{\alpha, k \alpha+1}^{k+1}\left(-\lambda_{0} t^{\alpha}\right), \quad n \geq 1,
\end{array}\right.
$$

where the sum is taken over the set

$$
\Theta_{n}^{k}=\left\{\left(k_{1}, k_{2}, \ldots, k_{n}\right): \sum_{j=1}^{n} k_{j}=k, \sum_{j=1}^{n} j k_{j}=n, k_{j} \in \mathbb{N}_{0}\right\} .
$$

An alternate expression for $p_{c}^{\alpha}(n, t)$ is obtained where the sum is taken over a slightly simplified set. It is observed that the CFPP is not a renewal process.

The paper is organized as follows: In Section 2, we set some notations and give some preliminary results related to Mittag-Leffler function and its generalizations, Bell polynomials etc. In Section 3, we introduce the CFPP and obtain its state probabilities. It is shown that the CFPP is a limiting case of a fractional counting process introduced and studied by Di Crescenzo et al. (2016). Its probability generating function (pgf), factorial moments, moment generating function (mgf), moments including mean and variance are derived. Also, it is shown that the CFPP is a compound fractional Poisson process with integer valued jumps of size $j \geq 1$. In Section 4 , we study a particular case of the CFPP, namely, the convoluted Poisson process (CPP). It is shown that the CPP is a Lévy process. Some subordination results related to CPP, CFPP and inverse $\alpha$-stable subordinator are obtained. In Section 5, we have shown that the CFPP exhibits the LRD property. Therefore, it has potential applications in ruin theory. It is also shown that the increments of CFPP have the SRD property.

\section{Preliminaries}

The set of integers is denoted by $\mathbb{Z}$, the set of positive integers by $\mathbb{N}$ and the set of non-negative integers by $\mathbb{N}_{0}$. The following definitions and known results related to discrete convolution, Bell polynomials, Mittag-Leffler function and its generalizations will be used.

2.1. Discrete convolution. The discrete convolution of two real-valued functions $f$ and $g$ whose support is the set of integers is defined as (see Damelin and Miller, 2012, p. 232)

$$
(f * g)(n):=\sum_{j=-\infty}^{\infty} f(j) g(n-j) .
$$

Here, $\sum_{j=-\infty}^{\infty}|f(j)|<\infty$ and $\sum_{j=-\infty}^{\infty}|g(j)|<\infty$, that is, $f, g \in \ell^{1}$ space.

2.2. Bell polynomials. The ordinary Bell polynomials $\hat{B}_{n, k}$ in $n-k+1$ variables is defined as

$$
\hat{B}_{n, k}\left(u_{1}, u_{2}, \ldots, u_{n-k+1}\right):=\sum_{\Lambda_{n}^{k}} k ! \prod_{j=1}^{n-k+1} \frac{u_{j}^{k_{j}}}{k_{j} !},
$$

where

$$
\Lambda_{n}^{k}=\left\{\left(k_{1}, k_{2}, \ldots, k_{n-k+1}\right): \sum_{j=1}^{n-k+1} k_{j}=k, \quad \sum_{j=1}^{n-k+1} j k_{j}=n, k_{j} \in \mathbb{N}_{0}\right\}
$$


The following results holds (see Comtet, 1974, pp. 133-137):

$$
\exp \left(x \sum_{j=1}^{\infty} u_{j} t^{j}\right)=1+\sum_{n=1}^{\infty} t^{n} \sum_{k=1}^{n} \hat{B}_{n, k}\left(u_{1}, u_{2}, \ldots, u_{n-k+1}\right) \frac{x^{k}}{k !}
$$

and

$$
\left(\sum_{j=1}^{\infty} u_{j} t^{j}\right)^{k}=\sum_{n=k}^{\infty} \hat{B}_{n, k}\left(u_{1}, u_{2}, \ldots, u_{n-k+1}\right) t^{n}
$$

2.3. Mittag-Leffler function and its generalizations. The Mellin-Barnes representation of the exponential function is given by (see Paris and Kaminski, 2001, Eq. (3.3.2))

$$
e^{x}=\frac{1}{2 \pi i} \int_{c-i \infty}^{c+i \infty} \Gamma(z)(-x)^{-z} \mathrm{~d} z, \quad x \neq 0
$$

where $i=\sqrt{-1}$.

The one-parameter Mittag-Leffler function is a generalization of the exponential function. It is defined as (see Mathai and Haubold, 2008)

$$
E_{\alpha, 1}(x):=\sum_{k=0}^{\infty} \frac{x^{k}}{\Gamma(k \alpha+1)}, \quad x \in \mathbb{R}
$$

where $\alpha>0$. For $\alpha=1$, it reduces to the exponential function. It is further generalized to two-parameter and three-parameter Mittag-Leffler functions.

The three-parameter Mittag-Leffler function is defined as

$$
E_{\alpha, \beta}^{\gamma}(x):=\frac{1}{\Gamma(\gamma)} \sum_{k=0}^{\infty} \frac{\Gamma(\gamma+k) x^{k}}{k ! \Gamma(k \alpha+\beta)}, \quad x \in \mathbb{R},
$$

where $\alpha>0, \beta>0$ and $\gamma>0$.

For $x \neq 0$, its Mellin-Barnes representation is given by (see Mathai and Haubold, 2008, Eq. (2.3.5))

$$
E_{\alpha, \beta}^{\gamma}(x)=\frac{1}{2 \pi i \Gamma(\gamma)} \int_{c-i \infty}^{c+i \infty} \frac{\Gamma(z) \Gamma(\gamma-z)}{\Gamma(\beta-\alpha z)}(-x)^{-z} \mathrm{~d} z,
$$

where $0<c<\gamma$. For $\gamma=1$, it reduces to two-parameter Mittag-Leffler function. Further, $\beta=\gamma=1$ reduce it to one-parameter Mittag-Leffler function. Note that (2.7) reduces to (2.5) for $\alpha=\beta=\gamma=1$.

Let $\alpha>0, \beta>0, t>0$ and $x, y$ be any two reals. The Laplace transform of the function $t^{\beta-1} E_{\alpha, \beta}^{\gamma}\left(\lambda t^{\alpha}\right)$ is given by (see Kilbas et al., 2006, Eq. (1.9.13)):

$$
\mathcal{L}\left\{t^{\beta-1} E_{\alpha, \beta}^{\gamma}\left(x t^{\alpha}\right) ; s\right\}=\frac{s^{\alpha \gamma-\beta}}{\left(s^{\alpha}-x\right)^{\gamma}}, s>|x|^{1 / \alpha} .
$$

The following result holds for one-parameter and three-parameter Mittag-Leffler functions (see Beghin and Orsingher, 2010, Eq. (2.30)):

$$
\sum_{k=0}^{\infty}\left(\lambda u t^{\alpha}\right)^{k} E_{\alpha, k \alpha+1}^{k+1}\left(-\lambda t^{\alpha}\right)=E_{\alpha, 1}\left(\lambda(u-1) t^{\alpha}\right), \quad|u| \leq 1 .
$$

Let $E_{\alpha, \beta}^{(n)}(\cdot)$ denote the $n$th derivative of two-parameter Mittag-Leffler function. Then, (see Kilbas et al., 2006, Eq. (1.9.5)):

$$
E_{\alpha, \beta}^{(n)}(x)=n ! E_{\alpha, n \alpha+\beta}^{n+1}(x), \quad n \geq 0 .
$$




\section{Convoluted fractional Poisson process}

Here, we introduce and study a process by varying intensity as a function of states and by taking discrete convolution in the governing difference-differential equation (1.1) of the TFPP. The introduced process is called the convoluted fractional Poisson process (CFPP) which we denote by $\left\{\mathcal{N}_{c}^{\alpha}(t)\right\}_{t \geq 0}, 0<\alpha \leq 1$. We define it as the stochastic process whose state probabilities $p_{c}^{\alpha}(n, t)=$ $\operatorname{Pr}\left\{\mathcal{N}_{c}^{\alpha}(t)=n\right\}$, satisfy

$$
\partial_{t}^{\alpha} p_{c}^{\alpha}(n, t)=-\lambda_{n} * p_{c}^{\alpha}(n, t)+\lambda_{n-1} * p_{c}^{\alpha}(n-1, t), \quad n \geq 0,
$$

with initial conditions

$$
p_{c}^{\alpha}(n, 0)= \begin{cases}1, & n=0 \\ 0, & n \geq 1\end{cases}
$$

and $p_{c}^{\alpha}(-n, t)=0$ for all $n \geq 1, t \geq 0$.

Here, $\left\{\lambda_{j}, j \in \mathbb{Z}\right\}$ is a sequence of intensity parameters such that $\lambda_{j}=0$ for all $j<0$ and $\lambda_{j}>\lambda_{j+1}>0$ for all $j \geq 0$ with $\lim _{j \rightarrow \infty} \lambda_{j+1} / \lambda_{j}<1$. Thus, it follows that

$$
\sum_{j=0}^{\infty}\left(\lambda_{j-1}-\lambda_{j}\right)=0
$$

as $\sum_{j=0}^{\infty} \lambda_{j}<\infty$ implies $\lambda_{j} \rightarrow 0$ as $j \rightarrow \infty$.

Using (2.1), the system of fractional differential equations (3.1) can be rewritten as

$$
\begin{aligned}
\partial_{t}^{\alpha} p_{c}^{\alpha}(n, t) & =-\sum_{j=0}^{n} \lambda_{j} p_{c}^{\alpha}(n-j, t)+\sum_{j=0}^{n-1} \lambda_{j} p_{c}^{\alpha}(n-j-1, t) \\
& =-\sum_{j=0}^{n} \lambda_{j} p_{c}^{\alpha}(n-j, t)+\sum_{j=1}^{n} \lambda_{j-1} p_{c}^{\alpha}(n-j, t) \\
& =-\lambda_{0} p_{c}^{\alpha}(n, t)+\sum_{j=1}^{n}\left(\lambda_{j-1}-\lambda_{j}\right) p_{c}^{\alpha}(n-j, t), \quad n \geq 0 .
\end{aligned}
$$

Remark 3.1. Recently, Di Crescenzo et al. (2016) studied a fractional counting process $\left\{M^{\alpha}(t)\right\}_{t \geq 0}$, $0<\alpha \leq 1$, which performs $k$ kinds of jumps of amplitude $1,2, \ldots, k$ with positive rates $\Lambda_{1}, \Lambda_{2}, \ldots$, $\Lambda_{k}$, respectively, where $k \in \mathbb{N}$ is fixed. Its state probabilities $q^{\alpha}(n, t)=\operatorname{Pr}\left\{M^{\alpha}(t)=n\right\}$ satisfy (see Di Crescenzo et al., 2016, Eq. (2.3))

$$
\partial_{t}^{\alpha} q^{\alpha}(n, t)=-\left(\Lambda_{1}+\Lambda_{2}+\cdots+\Lambda_{k}\right) q^{\alpha}(n, t)+\sum_{j=1}^{\min \{n, k\}} \Lambda_{j} q^{\alpha}(n-j, t), \quad n \geq 0,
$$

with

$$
q^{\alpha}(n, 0)= \begin{cases}1, & n=0 \\ 0, & n \geq 1\end{cases}
$$

For $k=1$, the system of fractional differential equation (3.4) reduces to (1.1). Thus, the TFPP follows as a particular case of $\left\{M^{\alpha}(t)\right\}_{t \geq 0}$. It is important to note that the CFPP is not a particular case of $\left\{M^{\alpha}(t)\right\}_{t \geq 0}$ for any choice of $k \in \mathbb{N}$. However, if we choose $\Lambda_{j}=\lambda_{j-1}-\lambda_{j}$, for all $j \geq 1$, then $\Lambda_{1}+\Lambda_{2}+\cdots+\Lambda_{k}=\lambda_{0}-\lambda_{k}$. As $\sum_{k=0}^{\infty} \lambda_{k}<\infty$ implies $\lambda_{k} \rightarrow 0$ as $k \rightarrow \infty$, the system (3.4) reduces to (3.3). Thus, the CFPP performs jumps of amplitude $j \geq 1$, and it is obtained as a limiting process of $\left\{M^{\alpha}(t)\right\}_{t \geq 0}$ by letting $k \rightarrow \infty$.

The following result gives the Laplace transform of the state probabilities of CFPP. 
Proposition 3.2. The Laplace transform of the state probabilities $\tilde{p}_{c}^{\alpha}(n, s), s>0$, of CFPP is given by

$$
\tilde{p}_{c}^{\alpha}(n, s)=\left\{\begin{array}{l}
\frac{s^{\alpha-1}}{s^{\alpha}+\lambda_{0}}, \quad n=0, \\
\sum_{k=1}^{n} \sum_{\Theta_{n}^{k}} k ! \prod_{j=1}^{n} \frac{\left(\lambda_{j-1}-\lambda_{j}\right)^{k_{j}}}{k_{j} !} \frac{s^{\alpha-1}}{\left(s^{\alpha}+\lambda_{0}\right)^{k+1}}, \quad n \geq 1,
\end{array}\right.
$$

where $\Theta_{n}^{k}=\left\{\left(k_{1}, k_{2}, \ldots, k_{n}\right): \sum_{j=1}^{n} k_{j}=k, \sum_{j=1}^{n} j k_{j}=n, k_{j} \in \mathbb{N}_{0}\right\}$.

Proof: On applying the Laplace transform in (3.3) and using (1.2), we get

$$
s^{\alpha} \tilde{p}_{c}^{\alpha}(n, s)-s^{\alpha-1} p_{c}^{\alpha}(n, 0)=-\lambda_{0} \tilde{p}_{c}^{\alpha}(n, s)+\sum_{m=1}^{n}\left(\lambda_{m-1}-\lambda_{m}\right) \tilde{p}_{c}^{\alpha}(n-m, s) .
$$

Thus,

$$
\tilde{p}_{c}^{\alpha}(n, s)=\left(s^{\alpha}+\lambda_{0}\right)^{-1}\left(\sum_{m=1}^{n}\left(\lambda_{m-1}-\lambda_{m}\right) \tilde{p}_{c}^{\alpha}(n-m, s)+s^{\alpha-1} p_{c}^{\alpha}(n, 0)\right) .
$$

Put $n=0$ in the above equation and use the initial conditions given in (3.1) to obtain

$$
\tilde{p}_{c}^{\alpha}(0, s)=\frac{s^{\alpha-1}}{s^{\alpha}+\lambda_{0}}
$$

So, the result holds for $n=0$. Next, we put $n=1$ in (3.6) to get

$$
\tilde{p}_{c}^{\alpha}(1, s)=\frac{\left(\lambda_{0}-\lambda_{1}\right) \tilde{p}(0, s)}{s^{\alpha}+\lambda_{0}}=\frac{\left(\lambda_{0}-\lambda_{1}\right) s^{\alpha-1}}{\left(s^{\alpha}+\lambda_{0}\right)^{2}}
$$

and the result holds for $n=1$. Now put $n=2$ in (3.6) to get

$$
\tilde{p}_{c}^{\alpha}(2, s)=\frac{\left(\lambda_{0}-\lambda_{1}\right)^{2} s^{\alpha-1}}{\left(s^{\alpha}+\lambda_{0}\right)^{3}}+\frac{\left(\lambda_{1}-\lambda_{2}\right) s^{\alpha-1}}{\left(s^{\alpha}+\lambda_{0}\right)^{2}} .
$$

Thus, the result holds for $n=2$. Assume the result (3.5) holds for $n=l$. From (3.6), we have

$$
\begin{aligned}
\tilde{p}_{c}^{\alpha}(l+1, s)= & \left(s^{\alpha}+\lambda_{0}\right)^{-1}\left(\sum_{m=1}^{l+1}\left(\lambda_{m-1}-\lambda_{m}\right) \tilde{p}_{c}^{\alpha}(l+1-m, s)\right) \\
= & \left(s^{\alpha}+\lambda_{0}\right)^{-1}\left(\sum_{m=1}^{l}\left(\lambda_{m-1}-\lambda_{m}\right) \tilde{p}_{c}^{\alpha}(l+1-m, s)\right)+\frac{\left(\lambda_{l}-\lambda_{l+1}\right) \tilde{p}_{c}^{\alpha}(0, s)}{\left(s^{\alpha}+\lambda_{0}\right)} \\
= & \sum_{m=1}^{l}\left(\lambda_{m-1}-\lambda_{m}\right) \sum_{k=1}^{l+1-m} \sum_{l+1-m}^{k} k ! \prod_{j=1}^{l+1-m} \frac{\left(\lambda_{j-1}-\lambda_{j}\right)^{k_{j}}}{k_{j} !} \frac{s^{\alpha-1}}{\left(s^{\alpha}+\lambda_{0}\right)^{k+2}} \\
& +\left(\lambda_{l}-\lambda_{l+1}\right) \frac{s^{\alpha-1}}{\left(s^{\alpha}+\lambda_{0}\right)^{2}} \\
= & \sum_{k=1}^{l+1} \sum_{\Theta_{l+1}^{k}} k ! \prod_{j=1}^{l+1} \frac{\left(\lambda_{j-1}-\lambda_{j}\right)^{k_{j}}}{k_{j} !} \frac{s^{\alpha-1}}{\left(s^{\alpha}+\lambda_{0}\right)^{k+1}} .
\end{aligned}
$$

Using the method of mathematical induction, the result (3.5) holds true for all $n \geq 0$.

The above result can be written in a different form by using the following result due to Kataria and Vellaisamy (2017a). 
Lemma 3.3. Let $e_{j}^{n}$ denotes the $n$-tuple vector with unity at the $j$ th place and zero elsewhere. Then

$$
\Theta_{n}^{k}=\left\{\sum_{j=1}^{n-k+1} k_{j} e_{j}^{n}:\left(k_{1}, k_{2}, \ldots, k_{n-k+1}\right) \in \Lambda_{n}^{k}\right\},
$$

where $\Lambda_{n}^{k}$ is given in (2.2).

Using the above result, an equivalent expression for $\tilde{p}_{c}^{\alpha}(n, s)$ is given by

$$
\tilde{p}_{c}^{\alpha}(n, s)=\left\{\begin{array}{l}
\frac{s^{\alpha-1}}{s^{\alpha}+\lambda_{0}}, \quad n=0 \\
\sum_{k=1}^{n} \sum_{\Lambda_{n}^{k}} k ! \prod_{j=1}^{n-k+1} \frac{\left(\lambda_{j-1}-\lambda_{j}\right)^{k_{j}}}{k_{j} !} \frac{s^{\alpha-1}}{\left(s^{\alpha}+\lambda_{0}\right)^{k+1}}, \quad n \geq 1 .
\end{array}\right.
$$
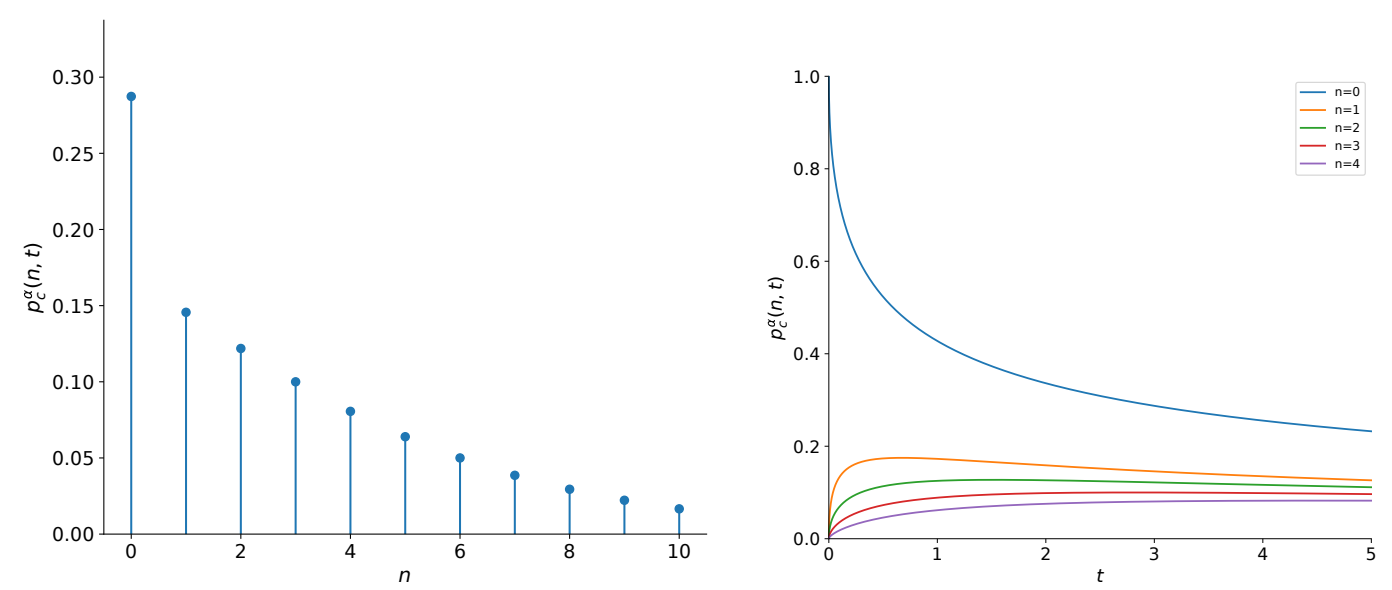

Figure 3.1. The figure on the left hand shows the probability distribution of CFPP for $\lambda_{j}=e^{-j}, j \geq 0, \alpha=0.5$ and $t=3$ whereas the figure on the right hand shows the probability distribution of CFPP for $0 \leq t \leq 5$.

Theorem 3.4. The one-dimensional distribution of CFPP is given by

$$
p_{c}^{\alpha}(n, t)=\left\{\begin{array}{l}
E_{\alpha, 1}\left(-\lambda_{0} t^{\alpha}\right), \quad n=0, \\
\sum_{k=1}^{n} \sum_{\Theta_{n}^{k}} k ! \prod_{j=1}^{n} \frac{\left(\lambda_{j-1}-\lambda_{j}\right)^{k_{j}}}{k_{j} !} t^{k \alpha} E_{\alpha, k \alpha+1}^{k+1}\left(-\lambda_{0} t^{\alpha}\right), \quad n \geq 1,
\end{array}\right.
$$

where $\Theta_{n}^{k}=\left\{\left(k_{1}, k_{2}, \ldots, k_{n}\right): \sum_{j=1}^{n} k_{j}=k, \quad \sum_{j=1}^{n} j k_{j}=n, k_{j} \in \mathbb{N}_{0}\right\}$.

Proof: Taking inverse Laplace transform in (3.5), we get

$$
\mathcal{L}^{-1}\left(\tilde{p}_{c}^{\alpha}(n, s) ; t\right)=\left\{\begin{array}{l}
\mathcal{L}^{-1}\left(\frac{s^{\alpha-1}}{s^{\alpha}+\lambda_{0}} ; t\right), \quad n=0, \\
\mathcal{L}^{-1}\left(\sum_{k=1}^{n} \sum_{\Theta_{n}^{k}} k ! \prod_{j=1}^{n} \frac{\left(\lambda_{j-1}-\lambda_{j}\right)^{k_{j}}}{k_{j} !} \frac{s^{\alpha-1}}{\left(s^{\alpha}+\lambda_{0}\right)^{k+1}} ; t\right), \quad n \geq 1 .
\end{array}\right.
$$

Using (2.8), the above equation reduces to (3.7). 
Using Lemma 3.3, we can rewrite (3.7) as

$$
p_{c}^{\alpha}(n, t)=\left\{\begin{array}{l}
E_{\alpha, 1}\left(-\lambda_{0} t^{\alpha}\right), \quad n=0 \\
\sum_{k=1}^{n} \sum_{\Lambda_{n}^{k}} k ! \prod_{j=1}^{n-k+1} \frac{\left(\lambda_{j-1}-\lambda_{j}\right)^{k_{j}}}{k_{j} !} t^{k \alpha} E_{\alpha, k \alpha+1}^{k+1}\left(-\lambda_{0} t^{\alpha}\right), \quad n \geq 1 .
\end{array}\right.
$$

Note that,

$$
\begin{aligned}
\sum_{n=0}^{\infty} p_{c}^{\alpha}(n, t) & =p_{c}^{\alpha}(0, t)+\sum_{n=1}^{\infty} p_{c}^{\alpha}(n, t) \\
& =E_{\alpha, 1}\left(-\lambda_{0} t^{\alpha}\right)+\sum_{n=1}^{\infty} \sum_{k=1}^{n} \sum_{\Lambda_{n}^{k}} k ! \prod_{j=1}^{n-k+1} \frac{\left(\lambda_{j-1}-\lambda_{j}\right)^{k_{j}}}{k_{j} !} t^{k \alpha} E_{\alpha, k \alpha+1}^{k+1}\left(-\lambda_{0} t^{\alpha}\right) \\
& =E_{\alpha, 1}\left(-\lambda_{0} t^{\alpha}\right)+\sum_{k=1}^{\infty} t^{k \alpha} E_{\alpha, k \alpha+1}^{k+1}\left(-\lambda_{0} t^{\alpha}\right) \sum_{n=k}^{\infty} \sum_{\Lambda_{n}^{k}} k ! \prod_{j=1}^{n-k+1} \frac{\left(\lambda_{j-1}-\lambda_{j}\right)^{k_{j}}}{k_{j} !} \\
& =E_{\alpha, 1}\left(-\lambda_{0} t^{\alpha}\right)+\sum_{k=1}^{\infty}\left(\sum_{j=1}^{\infty}\left(\lambda_{j-1}-\lambda_{j}\right)\right)^{k \alpha} E_{\alpha, k \alpha+1}^{k+1}\left(-\lambda_{0} t^{\alpha}\right), \quad(\text { using }(2.4)) \\
& =E_{\alpha, 1}\left(-\lambda_{0} t^{\alpha}\right)+\sum_{k=1}^{\infty}\left(\lambda_{0} t^{\alpha}\right)^{k} E_{\alpha, k \alpha+1}^{k+1}\left(-\lambda_{0} t^{\alpha}\right) \\
& =\sum_{k=0}^{\infty}\left(\lambda_{0} t^{\alpha}\right)^{k} E_{\alpha, k \alpha+1}^{k+1}\left(-\lambda_{0} t^{\alpha}\right)=1,
\end{aligned}
$$

where in the last step we have used (2.9). Thus, it follows that (3.7) is a valid distribution.

Remark 3.5. Let the random variable $W_{c}^{\alpha}$ be the waiting time of the first convoluted fractional Poisson event. Then, the distribution of $W_{c}^{\alpha}$ is given by

$$
\operatorname{Pr}\left\{W_{c}^{\alpha}>t\right\}=\operatorname{Pr}\left\{\mathcal{N}_{c}^{\alpha}(t)=0\right\}=E_{\alpha, 1}\left(-\lambda_{0} t^{\alpha}\right), t>0
$$

which coincides with the first waiting time of TFPP (see Beghin and Orsingher, 2009). However, the one-dimensional distributions of TFPP and CFPP differ. Thus, the fact that the TFPP is a renewal process (see Meerschaert et al., 2011) implies that the CFPP is not a renewal process.

The next result gives the pgf of CFPP.

Proposition 3.6. The pgf $G_{c}^{\alpha}(u, t)=\mathbb{E}\left(u^{\mathcal{N}_{c}^{\alpha}(t)}\right)$ of CFPP is given by

$$
G_{c}^{\alpha}(u, t)=E_{\alpha, 1}\left(\sum_{j=0}^{\infty} u^{j}\left(\lambda_{j-1}-\lambda_{j}\right) t^{\alpha}\right), \quad|u| \leq 1 .
$$


Proof: The Laplace transform of the pgf of CFPP can be obtained as follows:

$$
\begin{aligned}
& \tilde{G}_{c}^{\alpha}(u, s)=\int_{0}^{\infty} e^{-s t} G_{c}^{\alpha}(u, t) \mathrm{d} t, \quad s>0 \\
& =\int_{0}^{\infty} e^{-s t} \sum_{n=0}^{\infty} u^{n} p_{c}^{\alpha}(n, t) \mathrm{d} t \\
& =\int_{0}^{\infty} e^{-s t}\left(E_{\alpha, 1}\left(-\lambda_{0} t^{\alpha}\right)\right. \\
& \left.+\sum_{n=1}^{\infty} u^{n} \sum_{k=1}^{n} \sum_{\Lambda_{n}^{k}} k ! \prod_{j=1}^{n-k+1} \frac{\left(\lambda_{j-1}-\lambda_{j}\right)^{k_{j}}}{k_{j} !} t^{k \alpha} E_{\alpha, k \alpha+1}^{k+1}\left(-\lambda_{0} t^{\alpha}\right)\right) \mathrm{d} t \\
& =\frac{s^{\alpha-1}}{s^{\alpha}+\lambda_{0}}+\sum_{n=1}^{\infty} u^{n} \sum_{k=1}^{n} \sum_{\Lambda_{n}^{k}} k ! \prod_{j=1}^{n-k+1} \frac{\left(\lambda_{j-1}-\lambda_{j}\right)^{k_{j}}}{k_{j} !} \frac{s^{\alpha-1}}{\left(s^{\alpha}+\lambda_{0}\right)^{k+1}}, \quad(\text { using (2.8)) } \\
& =\frac{s^{\alpha-1}}{s^{\alpha}+\lambda_{0}}\left(1+\sum_{k=1}^{\infty} \frac{1}{\left(s^{\alpha}+\lambda_{0}\right)^{k}} \sum_{n=k}^{\infty} \sum_{\Lambda_{n}^{k}} k ! \prod_{j=1}^{n-k+1} \frac{\left(\lambda_{j-1}-\lambda_{j}\right)^{k_{j}}}{k_{j} !} u^{n}\right) \\
& =\frac{s^{\alpha-1}}{s^{\alpha}+\lambda_{0}}\left(1+\sum_{k=1}^{\infty}\left(\frac{\sum_{j=1}^{\infty} u^{j}\left(\lambda_{j-1}-\lambda_{j}\right)}{s^{\alpha}+\lambda_{0}}\right)^{k}\right), \quad(\operatorname{using}(2.4)) \\
& =\frac{s^{\alpha-1}}{s^{\alpha}+\lambda_{0}} \sum_{k=0}^{\infty}\left(\frac{\sum_{j=1}^{\infty} u^{j}\left(\lambda_{j-1}-\lambda_{j}\right)}{s^{\alpha}+\lambda_{0}}\right)^{k} \\
& =s^{\alpha-1}\left(s^{\alpha}-\sum_{j=0}^{\infty} u^{j}\left(\lambda_{j-1}-\lambda_{j}\right)\right)^{-1},
\end{aligned}
$$

which on using (2.8) gives (3.9).

Remark 3.7. The interchange of integral and sum in (3.10) can be justified by using the Fubini's theorem as follows:

$$
\begin{aligned}
\sum_{n=1}^{\infty} \int_{0}^{\infty} \mid & u^{n} \sum_{k=1}^{n} \sum_{\Lambda_{n}^{k}} k ! \prod_{j=1}^{n-k+1} \frac{\left(\lambda_{j-1}-\lambda_{j}\right)^{k_{j}}}{k_{j} !} e^{-s t} t^{k \alpha} E_{\alpha, k \alpha+1}^{k+1}\left(-\lambda_{0} t^{\alpha}\right) \mid \mathrm{d} t \\
& =\sum_{n=1}^{\infty}\left|u^{n}\right| \sum_{k=1}^{n} \sum_{\Lambda_{n}^{k}} k ! \prod_{j=1}^{n-k+1} \frac{\left(\lambda_{j-1}-\lambda_{j}\right)^{k_{j}}}{k_{j} !} \int_{0}^{\infty} e^{-s t} t^{k \alpha} E_{\alpha, k \alpha+1}^{k+1}\left(-\lambda_{0} t^{\alpha}\right) \mathrm{d} t \\
& \leq \sum_{n=1}^{\infty} \sum_{k=1}^{n} \sum_{\Lambda_{n}^{k}}^{k !} \prod_{j=1}^{n-k+1} \frac{\left(\lambda_{j-1}-\lambda_{j}\right)^{k_{j}}}{k_{j} !} \frac{s^{\alpha-1}}{\left(s^{\alpha}+\lambda_{0}\right)^{k+1}}, \quad(\text { using (2.8)) } \\
& =\frac{s^{\alpha-1}}{s^{\alpha}+\lambda_{0}} \sum_{k=1}^{\infty}\left(\frac{\lambda_{0}}{s^{\alpha}+\lambda_{0}}\right)^{k}, \quad(\text { using }(2.4)) \\
& =\frac{\lambda_{0}}{s\left(s^{\alpha}+\lambda_{0}\right)}<\infty .
\end{aligned}
$$


Remark 3.8. It is known that the Mittag-Leffler function is an eigenfunction of the Caputo fractional derivative. Thus, we have

$$
\partial_{t}^{\alpha} G_{c}^{\alpha}(u, t)=G_{c}^{\alpha}(u, t) \sum_{j=0}^{\infty} u^{j}\left(\lambda_{j-1}-\lambda_{j}\right), \quad G_{c}^{\alpha}(u, 0)=1 .
$$

If $\lambda_{j-1}-\lambda_{j}=\delta$ for all $j \geq 1$ then (3.12) reduces to

$$
\partial_{t}^{\alpha} G_{c}^{\alpha}(u, t)=G_{c}^{\alpha}(u, t)\left(\frac{\delta u}{1-u}-\lambda_{0}\right)
$$

Note that the Laplace transform of the pgf of CFPP can be also obtained as follows: On taking Laplace transform in (3.12), we get

$$
s^{\alpha} \tilde{G}_{c}^{\alpha}(u, s)-s^{\alpha-1} G_{c}^{\alpha}(u, 0)=\tilde{G}_{c}^{\alpha}(u, s) \sum_{j=0}^{\infty} u^{j}\left(\lambda_{j-1}-\lambda_{j}\right) .
$$

Thus,

$$
\tilde{G}_{c}^{\alpha}(u, s)=s^{\alpha-1}\left(s^{\alpha}-\sum_{j=0}^{\infty} u^{j}\left(\lambda_{j-1}-\lambda_{j}\right)\right)^{-1}
$$

which coincides with (3.11).

Next, we obtain the mean and variance of CFPP using its pgf. From (3.9), we have

$$
G_{c}^{\alpha}(u, t)=\sum_{k=0}^{\infty} \frac{t^{k \alpha}}{\Gamma(k \alpha+1)}\left(\sum_{j=0}^{\infty}\left(\lambda_{j-1}-\lambda_{j}\right) u^{j}\right)^{k} .
$$

Now, the mean of CFPP is given by

$$
\begin{aligned}
\mathbb{E}\left(\mathcal{N}_{c}^{\alpha}(t)\right) & =\left.\frac{\partial G_{c}^{\alpha}(u, t)}{\partial u}\right|_{u=1} \\
& =\sum_{k=1}^{\infty} \frac{k t^{k \alpha}}{\Gamma(k \alpha+1)}\left(\sum_{j=0}^{\infty}\left(\lambda_{j-1}-\lambda_{j}\right)\right)^{k-1}\left(\sum_{j=1}^{\infty}\left(\lambda_{j-1}-\lambda_{j}\right) j\right) \\
& =\frac{t^{\alpha}}{\Gamma(\alpha+1)} \sum_{j=0}^{\infty} \lambda_{j},
\end{aligned}
$$

where we have used (3.2) in the last step. Also, its variance can be obtained as follows:

$$
\begin{aligned}
\mathbb{E}\left(\mathcal{N}_{c}^{\alpha}(t)\left(\mathcal{N}_{c}^{\alpha}(t)-1\right)\right) & =\left.\frac{\partial^{2} G_{c}^{\alpha}(u, t)}{\partial u^{2}}\right|_{u=1} \\
& =\sum_{k=2}^{\infty} \frac{k(k-1) t^{k \alpha}}{\Gamma(k \alpha+1)}\left(\sum_{j=0}^{\infty}\left(\lambda_{j-1}-\lambda_{j}\right)\right)^{k-2}\left(\sum_{j=1}^{\infty}\left(\lambda_{j-1}-\lambda_{j}\right) j\right)^{2} \\
& +\sum_{k=1}^{\infty} \frac{k t^{k \alpha}}{\Gamma(k \alpha+1)}\left(\sum_{j=0}^{\infty}\left(\lambda_{j-1}-\lambda_{j}\right)\right)^{k-1}\left(\sum_{j=2}^{\infty}\left(\lambda_{j-1}-\lambda_{j}\right) j(j-1)\right) \\
& =\frac{2 t^{2 \alpha}}{\Gamma(2 \alpha+1)}\left(\sum_{j=0}^{\infty} \lambda_{j}\right)^{2}+\frac{2 t^{\alpha}}{\Gamma(\alpha+1)} \sum_{j=1}^{\infty} j \lambda_{j} .
\end{aligned}
$$


Thus,

$$
\mathbb{E}\left(\mathcal{N}_{c}^{\alpha}(t)^{2}\right)=\frac{2 t^{2 \alpha}}{\Gamma(2 \alpha+1)}\left(\sum_{j=0}^{\infty} \lambda_{j}\right)^{2}+\frac{2 t^{\alpha}}{\Gamma(\alpha+1)} \sum_{j=1}^{\infty} j \lambda_{j}+\frac{t^{\alpha}}{\Gamma(\alpha+1)} \sum_{j=0}^{\infty} \lambda_{j} .
$$

Hence, the variance of CFPP is given by

$$
\operatorname{Var}\left(\mathcal{N}_{c}^{\alpha}(t)\right)=\frac{t^{\alpha} \sum_{j=0}^{\infty} \lambda_{j}}{\Gamma(\alpha+1)}+\frac{2 t^{\alpha} \sum_{j=1}^{\infty} j \lambda_{j}}{\Gamma(\alpha+1)}+\frac{2\left(t^{\alpha} \sum_{j=0}^{\infty} \lambda_{j}\right)^{2}}{\Gamma(2 \alpha+1)}-\frac{\left(t^{\alpha} \sum_{j=0}^{\infty} \lambda_{j}\right)^{2}}{\Gamma^{2}(\alpha+1)}
$$

Remark 3.9. The mean and variance of $M^{\alpha}(t)$ is given by (see Di Crescenzo et al., 2016, Remark 2.11)

$$
\mathbb{E}\left(M^{\alpha}(t)\right)=\frac{t^{\alpha}}{\Gamma(\alpha+1)} \sum_{j=1}^{k} j \Lambda_{j}
$$

and

$$
\operatorname{Var}\left(M^{\alpha}(t)\right)=\frac{t^{\alpha}}{\Gamma(\alpha+1)} \sum_{j=1}^{k} j^{2} \Lambda_{j}+\frac{t^{2 \alpha}}{\alpha}\left(\frac{1}{\Gamma(2 \alpha)}-\frac{1}{\alpha \Gamma^{2}(\alpha)}\right)\left(\sum_{j=1}^{k} j \Lambda_{j}\right)^{2} .
$$

On taking $\Lambda_{j}=\lambda_{j-1}-\lambda_{j}, j \geq 1$ and letting $k \rightarrow \infty$, we get the mean and variance of CFPP.

The pgf of CFPP can also be utilized to obtain its factorial moments as follows:

Proposition 3.10. The rth factorial moment of the CFPP $\psi_{c}^{\alpha}(r, t)=\mathbb{E}\left(\mathcal{N}_{c}^{\alpha}(t)\left(\mathcal{N}_{c}^{\alpha}(t)-1\right) \cdots\right.$ $\left.\left(\mathcal{N}_{c}^{\alpha}(t)-r+1\right)\right), r \geq 1$, is given by

$$
\psi_{c}^{\alpha}(r, t)=r ! \sum_{k=1}^{r} \frac{t^{k \alpha}}{\Gamma(k \alpha+1)} \sum_{\substack{\sum_{j=1}^{k} m_{j}=r \\ m_{j} \in \mathbb{N}}} \prod_{\ell=1}^{k}\left(\frac{1}{m_{\ell} !} \sum_{j=0}^{\infty}(j)_{m_{\ell}}\left(\lambda_{j-1}-\lambda_{j}\right)\right),
$$

where $(j)_{m_{\ell}}=j(j-1) \ldots\left(j-m_{\ell}+1\right)$ denotes the falling factorial.

Proof: From (3.9), we get

$$
\begin{aligned}
\psi_{c}^{\alpha}(r, t) & =\left.\frac{\partial^{r} G_{c}^{\alpha}(u, t)}{\partial u^{r}}\right|_{u=1} \\
& =\left.\sum_{k=0}^{r} \frac{1}{k !} E_{\alpha, 1}^{(k)}\left(t^{\alpha} \sum_{j=0}^{\infty}\left(\lambda_{j-1}-\lambda_{j}\right) u^{j}\right) A_{r, k}\left(t^{\alpha} \sum_{j=0}^{\infty}\left(\lambda_{j-1}-\lambda_{j}\right) u^{j}\right)\right|_{u=1},
\end{aligned}
$$

where we have used the $r$ th derivative of composition of two functions (see Johnson, 2002, Eq. (3.3)). Here,

$$
\begin{aligned}
& \left.A_{r, k}\left(t^{\alpha} \sum_{j=0}^{\infty}\left(\lambda_{j-1}-\lambda_{j}\right) u^{j}\right)\right|_{u=1} \\
& \quad=\left.\sum_{m=0}^{k} \frac{k !}{m !(k-m) !}\left(-t^{\alpha} \sum_{j=0}^{\infty}\left(\lambda_{j-1}-\lambda_{j}\right) u^{j}\right)^{k-m} \frac{\mathrm{d}^{r}}{\mathrm{~d} u^{r}}\left(t^{\alpha} \sum_{j=0}^{\infty}\left(\lambda_{j-1}-\lambda_{j}\right) u^{j}\right)^{m}\right|_{u=1} \\
& \quad=\left.t^{k \alpha} \frac{\mathrm{d}^{r}}{\mathrm{~d} u^{r}}\left(\sum_{j=0}^{\infty}\left(\lambda_{j-1}-\lambda_{j}\right) u^{j}\right)^{k}\right|_{u=1}
\end{aligned}
$$


where the last step follows by using (3.2). From (2.10), we get

$$
\begin{aligned}
\left.E_{\alpha, 1}^{(k)}\left(t^{\alpha} \sum_{j=0}^{\infty}\left(\lambda_{j-1}-\lambda_{j}\right) u^{j}\right)\right|_{u=1} & =\left.k ! E_{\alpha, k \alpha+1}^{k+1}\left(t^{\alpha} \sum_{j=0}^{\infty}\left(\lambda_{j-1}-\lambda_{j}\right) u^{j}\right)\right|_{u=1} \\
& =\frac{k !}{\Gamma(k \alpha+1)}
\end{aligned}
$$

Now, by using the following result (see Johnson, 2002, Eq. (3.6))

$$
\frac{\mathrm{d}^{r}}{\mathrm{~d} w^{r}}(f(w))^{k}=\sum_{\substack{m_{1}+m_{2}+\cdots+m_{k}=r \\ m_{j} \in \mathbb{N}_{0}}} \frac{r !}{m_{1} ! m_{2} ! \ldots m_{k} !} f^{\left(m_{1}\right)}(w) f^{\left(m_{2}\right)}(w) \ldots f^{\left(m_{k}\right)}(w),
$$

we get

$$
\begin{aligned}
\left.\frac{\mathrm{d}^{r}}{\mathrm{~d} u^{r}}\left(\sum_{j=0}^{\infty}\left(\lambda_{j-1}-\lambda_{j}\right) u^{j}\right)^{k}\right|_{u=1} & =\left.r ! \sum_{\substack{\sum_{j=1}^{k} m_{j}=r \\
m_{j} \in \mathbb{N}_{0}}} \prod_{\ell=1}^{k} \frac{1}{m_{\ell} !} \frac{\mathrm{d}^{m_{\ell}}}{\mathrm{d} u^{m_{\ell}}}\left(\sum_{j=0}^{\infty}\left(\lambda_{j-1}-\lambda_{j}\right) u^{j}\right)\right|_{u=1} \\
& =r ! \sum_{\substack{\sum_{j=1}^{k} m_{j}=r \\
m_{j} \in \mathbb{N}}} \prod_{\ell=1}^{k} \frac{1}{m_{\ell} !} \sum_{j=0}^{\infty}(j)_{m_{\ell}}\left(\lambda_{j-1}-\lambda_{j}\right) .
\end{aligned}
$$

Note that the expression in right hand side of (3.19) vanishes for $k=0$. Finally, on substituting (3.16), (3.17) and (3.19) in (3.15), we get the required result.

The next result gives the mgf of CFPP on non-positive support which is obtained by substituting $u=e^{-\omega}$ in (3.9).

Proposition 3.11. The $\operatorname{mgf} m_{c}^{\alpha}(w, t)=\mathbb{E}\left(e^{-w \mathcal{N}_{c}^{\alpha}(t)}\right), w \geq 0$, of CFPP is given by

$$
m_{c}^{\alpha}(w, t)=E_{\alpha, 1}\left(t^{\alpha} \sum_{j=0}^{\infty}\left(\lambda_{j-1}-\lambda_{j}\right) e^{-w j}\right)
$$

The mgf of CFPP solves the following fractional differential equation:

$$
\partial_{t}^{\alpha} m_{c}^{\alpha}(w, t)=m_{c}^{\alpha}(w, t) \sum_{j=0}^{\infty} e^{-w j}\left(\lambda_{j-1}-\lambda_{j}\right), \quad m_{c}^{\alpha}(w, 0)=1 .
$$

The above equation can be solved by using the Laplace transform method to obtain the mgf (3.20). The mean and variance of the CFPP can also be obtained from its mgf.

Proposition 3.12. The rth moment $\mu_{c}^{\alpha}(r, t)=\mathbb{E}\left(\left(\mathcal{N}_{c}^{\alpha}(t)\right)^{r}\right), r \geq 1$, of CFPP is given by

$$
\mu_{c}^{\alpha}(r, t)=r ! \sum_{k=1}^{r} \frac{t^{k \alpha}}{\Gamma(k \alpha+1)} \sum_{\substack{\sum_{j=1}^{k} m_{j}=r \\ m_{j} \in \mathbb{N}}} \prod_{\ell=1}^{k}\left(\frac{1}{m_{\ell} !} \sum_{j=0}^{\infty} j^{m_{\ell}}\left(\lambda_{j-1}-\lambda_{j}\right)\right) .
$$


Proof: Taking the $r$ th derivative of composition of two functions (see Johnson, 2002, Eq. (3.3)), we get

$$
\begin{aligned}
\mu_{c}^{\alpha}(r, t) & =\left.(-1)^{r} \frac{\partial^{r} m_{c}^{\alpha}(w, t)}{\partial w^{r}}\right|_{w=0} \\
& =\left.\sum_{k=0}^{r} \frac{(-1)^{r}}{k !} E_{\alpha, 1}^{(k)}\left(t^{\alpha} \sum_{j=0}^{\infty}\left(\lambda_{j-1}-\lambda_{j}\right) e^{-w j}\right) B_{r, k}\left(t^{\alpha} \sum_{j=0}^{\infty}\left(\lambda_{j-1}-\lambda_{j}\right) e^{-w j}\right)\right|_{w=0},
\end{aligned}
$$

where

$$
\left.B_{r, k}\left(t^{\alpha} \sum_{j=0}^{\infty}\left(\lambda_{j-1}-\lambda_{j}\right) e^{-w j}\right)\right|_{w=0}=\left.t^{k \alpha} \frac{\mathrm{d}^{r}}{\mathrm{~d} w^{r}}\left(\sum_{j=0}^{\infty}\left(\lambda_{j-1}-\lambda_{j}\right) e^{-w j}\right)^{k}\right|_{w=0}
$$

Now, from (2.10), we get

$$
\left.E_{\alpha, 1}^{(k)}\left(t^{\alpha} \sum_{j=0}^{\infty}\left(\lambda_{j-1}-\lambda_{j}\right) e^{-w j}\right)\right|_{w=0}=\frac{k !}{\Gamma(k \alpha+1)} .
$$

Using (3.18), we have

$$
\left.\frac{\mathrm{d}^{r}}{\mathrm{~d} w^{r}}\left(\sum_{j=0}^{\infty}\left(\lambda_{j-1}-\lambda_{j}\right) e^{-w j}\right)^{k}\right|_{w=0}=(-1)^{r} r ! \sum_{\substack{\sum_{j=1}^{k} m_{j}=r \\ m_{j} \in \mathbb{N}}} \prod_{\ell=1}^{k} \frac{1}{m_{\ell} !} \sum_{j=0}^{\infty} j^{m_{\ell}}\left(\lambda_{j-1}-\lambda_{j}\right) .
$$

The result follows on substituting (3.22)-(3.24) in (3.21).

In the following result it is shown that the CFPP is equal in distribution to a compound fractional Poisson process. Thus, it is neither Markovian nor a Lévy process.

Theorem 3.13. Let $\left\{N^{\alpha}(t)\right\}_{t \geq 0}, 0<\alpha \leq 1$, be the TFPP with intensity parameter $\lambda_{0}>0$ and $\left\{X_{i}\right\}_{i \geq 1}$ be a sequence of independent and identically distributed (iid) random variables with the following distribution:

$$
\operatorname{Pr}\left\{X_{1}=j\right\}=\frac{\lambda_{j-1}-\lambda_{j}}{\lambda_{0}}, \quad j \geq 1
$$

Then,

$$
\mathcal{N}_{c}^{\alpha}(t) \stackrel{d}{=} \sum_{i=1}^{N^{\alpha}(t)} X_{i}, \quad t \geq 0,
$$

where $\left\{X_{i}\right\}_{i \geq 1}$ is independent of $\left\{N^{\alpha}(t)\right\}_{t \geq 0}$.

Proof: The mgf of $N^{\alpha}(t), t \geq 0$, is given by (see Laskin, 2003, Eq. (35))

$$
\mathbb{E}\left(e^{-w N^{\alpha}(t)}\right)=E_{\alpha, 1}\left(\lambda_{0} t^{\alpha}\left(e^{-w}-1\right)\right), \quad w \geq 0 .
$$

Also, the mgf of $X_{i}, i \geq 1$, can be obtained as

$$
\mathbb{E}\left(e^{-w X_{i}}\right)=\frac{1}{\lambda_{0}} \sum_{j=1}^{\infty}\left(\lambda_{j-1}-\lambda_{j}\right) e^{-j w} .
$$


Now,

$$
\begin{aligned}
\mathbb{E}\left(e^{-w \sum_{i=1}^{N^{\alpha}(t)} X_{i}}\right) & =\mathbb{E}\left(\mathbb{E}\left(e^{-w \sum_{i=1}^{N^{\alpha}(t)} X_{i}} \mid N^{\alpha}(t)\right)\right) \\
& =\mathbb{E}\left(\left(\mathbb{E}\left(e^{-w X_{1}}\right)\right)^{N^{\alpha}(t)}\right) \\
& =\mathbb{E}\left(\exp \left(N^{\alpha}(t) \ln \left(\frac{1}{\lambda_{0}} \sum_{j=1}^{\infty}\left(\lambda_{j-1}-\lambda_{j}\right) e^{-j w}\right)\right)\right), \quad(\operatorname{using}(3.27)) \\
& =E_{\alpha, 1}\left(\lambda_{0} t^{\alpha}\left(\exp \left(\ln \frac{1}{\lambda_{0}} \sum_{j=1}^{\infty}\left(\lambda_{j-1}-\lambda_{j}\right) e^{-w j}\right)-1\right)\right) \\
& =E_{\alpha, 1}\left(t^{\alpha} \sum_{j=0}^{\infty}\left(\lambda_{j-1}-\lambda_{j}\right) e^{-w j}\right)
\end{aligned}
$$

which agrees with the mgf of CFPP (3.20). This completes the proof.

In view of the above result, the CFPP can be regarded as a special case of the compound fractional Poisson process $\sum_{i=1}^{N^{\alpha}(t)} X_{i}$ (see Beghin and Macci, 2014, Eq. (7)) when the probability mass function $q_{j}=\operatorname{Pr}\left\{X_{1}=j\right\}$ for all integers $j \geq 1$ is given by (3.25) and the intensity parameter of TFPP is $\lambda_{0}>0$. Thus, on substituting $\lambda=\lambda_{0}$ and $q_{j}=\left(\lambda_{j-1}-\lambda_{j}\right) / \lambda_{0}$ in Eq. (12) of Beghin and Macci (2014), we get the governing system of fractional differential equations (3.3) for the CFPP. It is known that the compound fractional Poisson process has applications in ruin theory. Biard and Saussereau (2014) used it to model the claims received by an insurance company. As the CFPP is a version of the compound fractional Poisson process with the jumps of amplitude $j \geq 1$, it can be used in ruin theory to model the claims of any size. For example, in the case of catastrophic events like earthquake or tsunami, the number of destroyed houses or property can be of arbitrary magnitude.

Remark 3.14. The following result holds for $M^{\alpha}(t)$ (see Di Crescenzo et al., 2016, Proposition 2.2): Let $\Lambda_{j}>0$ for all $1 \leq j \leq k$ and $\left\{N^{\alpha}(t)\right\}_{t \geq 0}, 0<\alpha \leq 1$, be the TFPP with intensity parameter $\Lambda=\Lambda_{1}+\Lambda_{2}+\cdots+\Lambda_{k}$. Also, let $\left\{X_{i}\right\}_{i \geq 1}$ be a sequence of iid random variables such that it is independent of $\left\{N^{\alpha}(t)\right\}_{t \geq 0}$ and

$$
\operatorname{Pr}\left\{X_{i}=j\right\}=\frac{\Lambda_{j}}{\Lambda_{1}+\Lambda_{2}+\cdots+\Lambda_{k}}, \quad j=1,2, \ldots, k .
$$

Then,

$$
M^{\alpha}(t) \stackrel{d}{=} \sum_{i=1}^{N^{\alpha}(t)} X_{i}, \quad t \geq 0 .
$$

On taking $\Lambda_{j}=\lambda_{j-1}-\lambda_{j}, j \geq 1$ and letting $k \rightarrow \infty$, we get $\Lambda=\lambda_{0}$. Thus, Theorem 3.13 follows as the limiting case of Proposition 2.2, Di Crescenzo et al. (2016).

Remark 3.15. The one-dimensional distribution of TFPP is given by

$$
\operatorname{Pr}\left\{N^{\alpha}(t)=n\right\}=\left(\lambda_{0} t^{\alpha}\right)^{n} E_{\alpha, n \alpha+1}^{n+1}\left(-\lambda_{0} t^{\alpha}\right), \quad n \geq 0 .
$$

For $n=0$, using (3.26) we have

$$
\operatorname{Pr}\left\{\mathcal{N}_{c}^{\alpha}(t)=0\right\}=\operatorname{Pr}\left\{N^{\alpha}(t)=0\right\}=E_{\alpha, 1}\left(-\lambda_{0} t^{\alpha}\right) .
$$


As $X_{i}$ 's are independent of $N^{\alpha}(t)$, for $n \geq 1$, we get

$$
\begin{aligned}
\operatorname{Pr}\left\{\mathcal{N}_{c}^{\alpha}(t)=n\right\} & =\sum_{k=1}^{n} \operatorname{Pr}\left\{X_{1}+X_{2}+\cdots+X_{k}=n\right\} \operatorname{Pr}\left\{N^{\alpha}(t)=k\right\} \\
& =\sum_{k=1}^{n} \sum_{\Theta_{n}^{k}} k ! \prod_{j=1}^{n} \frac{\left(\lambda_{j-1}-\lambda_{j}\right)^{k_{j}}}{k_{j} !} t^{k \alpha} E_{\alpha, k \alpha+1}^{k+1}\left(-\lambda_{0} t^{\alpha}\right),
\end{aligned}
$$

where $k_{j}$ is the total number of claims of $j$ units. The above expression agrees with (3.7).

As $X_{i}$ 's are iid, we have

$$
\begin{aligned}
\operatorname{Pr}\left\{X_{1}+X_{2}+\cdots+X_{k}=n\right\} & =\sum_{\substack{m_{1}+m_{2}+\cdots+m_{k}=n \\
m_{j} \in \mathbb{N}}} \operatorname{Pr}\left\{X_{1}=m_{1}, X_{2}=m_{2}, \ldots, X_{k}=m_{k}\right\} \\
& =\sum_{\substack{m_{1}+m_{2}+\cdots+m_{k}=n \\
m_{j} \in \mathbb{N}}} \prod_{j=1}^{k} \operatorname{Pr}\left\{X_{j}=m_{j}\right\} \\
& =\sum_{\substack{m_{1}+m_{2}+\cdots+m_{k}=n \\
m_{j} \in \mathbb{N}}} \frac{1}{\lambda_{0}^{k}} \prod_{j=1}^{k}\left(\lambda_{m_{j}-1}-\lambda_{m_{j}}\right),
\end{aligned}
$$

where we have used (3.25). Substituting (3.29) in (3.28), we get an equivalent expression for the one-dimensional distribution of the CFPP as

$$
\operatorname{Pr}\left\{\mathcal{N}_{c}^{\alpha}(t)=n\right\}=\sum_{k=1}^{n} \sum_{\substack{m_{2}+\cdots+m_{k} \\ m_{j} \in \mathbb{N}}} \prod_{j=1}^{k}\left(\lambda_{m_{j}-1}-\lambda_{m_{j}}\right) t^{k \alpha} E_{\alpha, k \alpha+1}^{k+1}\left(-\lambda_{0} t^{\alpha}\right) .
$$

\section{Convoluted Poisson process: A special case of CFPP}

Here, we discuss a particular case of the CFPP, namely, the convoluted Poisson process (CPP) which we denote by $\left\{\mathcal{N}_{c}(t)\right\}_{t \geq 0}$.

The CPP is defined as a stochastic process whose state probabilities satisfy

$$
\frac{\mathrm{d}}{\mathrm{d} t} p_{c}(n, t)=-\lambda_{n} * p_{c}(n, t)+\lambda_{n-1} * p_{c}(n-1, t),
$$

with $p_{c}(n, 0)=\delta_{0}(n), n \geq 0$. The conditions on $\lambda_{n}$ 's are same as in the case of CFPP.

For $\alpha=1$, the CFPP reduces to CPP. Thus, its state probabilities are given by

$$
p_{c}(n, t)=\left\{\begin{array}{l}
e^{-\lambda_{0} t}, \quad n=0 \\
\sum_{k=1}^{n} \sum_{\Lambda_{n}^{k}} \prod_{j=1}^{n-k+1} \frac{\left(\lambda_{j-1}-\lambda_{j}\right)^{k_{j}}}{k_{j} !} t^{k} e^{-\lambda_{0} t}, \quad n \geq 1 .
\end{array}\right.
$$

Remark 4.1. Let $W_{c}$ be the first waiting time of CPP. Then,

$$
\operatorname{Pr}\left\{W_{c}>t\right\}=\operatorname{Pr}\left\{\mathcal{N}_{c}(t)=0\right\}=e^{-\lambda_{0} t}, t>0,
$$

which coincides with the first waiting time of Poisson process. It is known that the Poisson process is a renewal process. Thus, it implies that the CPP is not a renewal process as the one-dimensional distributions of Poisson process and CPP are different. 

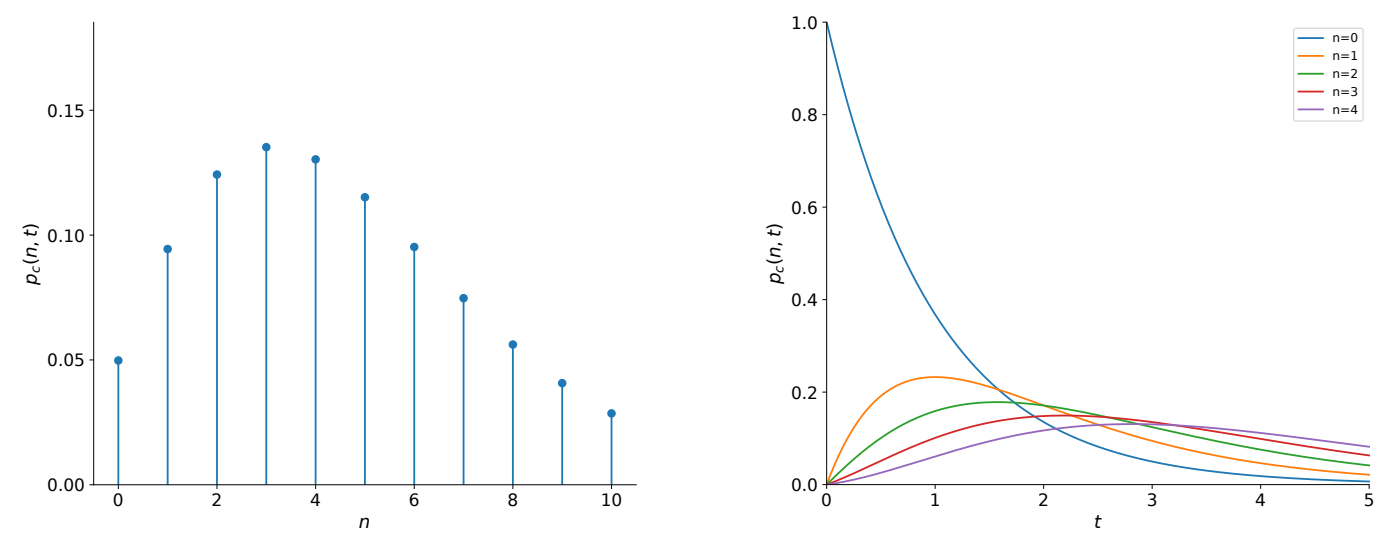

FIgURE 4.2. The figure on the left hand shows the probability distribution of CPP for $\lambda_{j}=e^{-j}, j \geq 0$ and $t=3$ whereas the figure on the right hand shows the probability distribution of CPP for $0 \leq t \leq 5$.

A direct method to obtain the pgf of CPP is as follows:

$$
\begin{aligned}
G_{c}(u, t) & =p_{c}(0, t)+\sum_{n=1}^{\infty} u^{n} p_{c}(n, t) \\
& =e^{-t \lambda_{0}}+\sum_{n=1}^{\infty} u^{n} \sum_{k=1}^{n} \sum_{\Lambda_{n}^{k}} \prod_{j=1}^{n-k+1} \frac{\left(\lambda_{j-1}-\lambda_{j}\right)^{k_{j}}}{k_{j} !} t^{k} e^{-t \lambda_{0}} \\
& =e^{-t \lambda_{0}}\left(1+\sum_{n=1}^{\infty} \sum_{k=1}^{n} \sum_{\Lambda_{n}^{k}} \prod_{j=1}^{n-k+1} \frac{\left(\lambda_{j-1}-\lambda_{j}\right)^{k_{j}}}{k_{j} !} u^{n} t^{k}\right) \\
& =e^{-t \lambda_{0}} \exp \left(t \sum_{j=1}^{\infty} u^{j}\left(\lambda_{j-1}-\lambda_{j}\right)\right), \quad(\operatorname{using}(2.3)) \\
& =\exp \left(t \sum_{j=0}^{\infty} u^{j}\left(\lambda_{j-1}-\lambda_{j}\right)\right) .
\end{aligned}
$$

Also, its mean and variance are given by

$$
\mathbb{E}\left(\mathcal{N}_{c}(t)\right)=t \sum_{j=0}^{\infty} \lambda_{j}
$$

and

$$
\operatorname{Var}\left(\mathcal{N}_{c}(t)\right)=t\left(\sum_{j=0}^{\infty} \lambda_{j}+2 \sum_{j=1}^{\infty} j \lambda_{j}\right)
$$

respectively.

Remark 4.2. The CPP exhibits overdispersion as

$$
\operatorname{Var}\left(\mathcal{N}_{c}(t)\right)-\mathbb{E}\left(\mathcal{N}_{c}(t)\right)=2 t \sum_{j=1}^{\infty} j \lambda_{j}>0
$$

for $t>0$.

Theorem 4.3. The CPP is a Lévy process. 
Proof: The characteristic function $\phi_{c}(\xi, t)$ of $\mathrm{CPP}$ is given by

$$
\begin{aligned}
\phi_{c}(\xi, t)=\mathbb{E}\left(e^{i \xi \mathcal{N}_{c}(t)}\right) & =\sum_{n=0}^{\infty} e^{i \xi n} p_{c}(n, t), \quad \xi \in \mathbb{R} \\
& =e^{-t \lambda_{0}}\left(1+\sum_{n=1}^{\infty} e^{i \xi n} \sum_{k=1}^{n} \sum_{\Lambda_{n}^{k}} \prod_{j=1}^{n-k+1} \frac{\left(\lambda_{j-1}-\lambda_{j}\right)^{k_{j}}}{k_{j} !} t^{k}\right) \\
& =e^{-t \lambda_{0}} \exp \left(t \sum_{j=1}^{\infty} e^{i \xi j}\left(\lambda_{j-1}-\lambda_{j}\right)\right), \quad(\text { using }(2.3)) \\
& =\exp \left(-t \sum_{j=0}^{\infty} e^{i \xi j}\left(\lambda_{j}-\lambda_{j-1}\right)\right) .
\end{aligned}
$$

So, its characteristic exponent is

$$
\psi(\xi)=\sum_{j=0}^{\infty}\left(\lambda_{j}-\lambda_{j-1}\right) e^{i \xi j}=\sum_{j=0}^{\infty}\left(\lambda_{j-1}-\lambda_{j}\right)\left(1-e^{i \xi j}\right) .
$$

Thus, the CPP is a Lévy process with Lévy measure $\Pi(\mathrm{d} x)=\sum_{j=0}^{\infty}\left(\lambda_{j-1}-\lambda_{j}\right) \delta_{j} \mathrm{~d} x$, where $\delta_{j}$ 's are Dirac measures.

Remark 4.4. Substituting $\alpha=1$ in (3.26), we get

$$
\mathcal{N}_{c}(t) \stackrel{d}{=} \sum_{i=1}^{N(t)} X_{i}, \quad t \geq 0
$$

where $\left\{X_{i}\right\}_{i \geq 1}$ is independent of the Poisson process $\{N(t)\}_{t \geq 0}$, i.e., the CPP is a compound Poisson process. Hence, it's a Lévy process.

Let $\left\{T_{2 \alpha}(t)\right\}_{t>0}$ be a random process whose distribution is given by the folded solution of following fractional diffusion equation (see Orsingher and Beghin, 2004):

$$
\partial_{t}^{2 \alpha} u(x, t)=\frac{\partial^{2}}{\partial x^{2}} u(x, t), \quad x \in \mathbb{R}, t>0,
$$

with $u(x, 0)=\delta(x)$ for $0<\alpha \leq 1$ and $\frac{\partial}{\partial t} u(x, 0)=0$ for $1 / 2<\alpha \leq 1$.

The Laplace transform of the folded solution $f_{T_{2 \alpha}}(x, t)$ of $(4.2)$ is given by (see Orsingher and Polito, 2010, Eq. (2.29))

$$
\int_{0}^{\infty} e^{-s t} f_{T_{2 \alpha}}(x, t) \mathrm{d} t=s^{\alpha-1} e^{-x s^{\alpha}}, \quad x>0 .
$$

The next result establish a time-changed relationship between the CPP and CFPP.

Theorem 4.5. Let the process $\left\{T_{2 \alpha}(t)\right\}_{t>0}, 0<\alpha \leq 1$, be independent of the CPP $\left\{\mathcal{N}_{c}(t)\right\}_{t>0}$. Then,

$$
\mathcal{N}_{c}^{\alpha}(t) \stackrel{d}{=} \mathcal{N}_{c}\left(T_{2 \alpha}(t)\right)
$$


Proof: From (3.10), we have

$$
\begin{aligned}
\tilde{G}_{c}^{\alpha}(u, s) & =\frac{s^{\alpha-1}}{s^{\alpha}+\lambda_{0}}+\sum_{n=1}^{\infty} u^{n} \sum_{k=1}^{n} \sum_{\Lambda_{n}^{k}} k ! \prod_{j=1}^{n-k+1} \frac{\left(\lambda_{j-1}-\lambda_{j}\right)^{k_{j}}}{k_{j} !} \frac{s^{\alpha-1}}{\left(s^{\alpha}+\lambda_{0}\right)^{k+1}}, \quad s>0 \\
& =\int_{0}^{\infty} s^{\alpha-1}\left(e^{-\mu\left(s^{\alpha}+\lambda_{0}\right)}+\sum_{n=1}^{\infty} u^{n} \sum_{k=1}^{n} \sum_{\Lambda_{n}^{k}} \prod_{j=1}^{n-k+1} \frac{\left(\lambda_{j-1}-\lambda_{j}\right)^{k_{j}}}{k_{j} !} e^{-\mu\left(s^{\alpha}+\lambda_{0}\right)} \mu^{k}\right) \mathrm{d} \mu \\
& =\int_{0}^{\infty} s^{\alpha-1} e^{-\mu s^{\alpha}}\left(e^{-\mu \lambda_{0}}+\sum_{n=1}^{\infty} u^{n} \sum_{k=1}^{n} \sum_{\Lambda_{n}^{k}} \prod_{j=1}^{n-k+1} \frac{\left(\lambda_{j-1}-\lambda_{j}\right)^{k_{j}}}{k_{j} !} e^{-\mu \lambda_{0}} \mu^{k}\right) \mathrm{d} \mu \\
& =\int_{0}^{\infty} s^{\alpha-1} e^{-\mu s^{\alpha}} G_{c}(u, \mu) \mathrm{d} \mu, \quad(\text { using }(4.1)) \\
& =\int_{0}^{\infty} G_{c}(u, \mu) \int_{0}^{\infty} e^{-s t} f_{T_{2 \alpha}}(\mu, t) \mathrm{d} t \mathrm{~d} \mu, \quad(u \operatorname{sing}(4.3)) \\
& =\int_{0}^{\infty} e^{-s t}\left(\int_{0}^{\infty} G_{c}(u, \mu) f_{T_{2 \alpha}}(\mu, t) \mathrm{d} \mu\right) \mathrm{d} t .
\end{aligned}
$$

By uniqueness of Laplace transform, we get

$$
G_{c}^{\alpha}(u, t)=\int_{0}^{\infty} G_{c}(u, \mu) f_{T_{2 \alpha}}(\mu, t) \mathrm{d} \mu .
$$

This completes the proof.

Remark 4.6. For $\alpha=1 / 2$, the process $\left\{T_{2 \alpha}(t)\right\}_{t>0}$ is equal in distribution to the reflecting Brownian motion $\{|B(t)|\}_{t>0}$ as the diffusion equation (4.2) reduces to the heat equation

$$
\left\{\begin{array}{l}
\frac{\partial}{\partial t} u(x, t)=\frac{\partial^{2}}{\partial x^{2}} u(x, t), \quad x \in \mathbb{R}, t>0, \\
u(x, 0)=\delta(x) .
\end{array}\right.
$$

So, $\mathcal{N}_{c}^{1 / 2}(t)$ coincides with CPP at a Brownian time, that is, $\mathcal{N}_{c}(|B(t)|), t>0$.

Remark 4.7. Let $\left\{H^{\alpha}(t)\right\}_{t>0}, 0<\alpha<1$, be an inverse $\alpha$-stable subordinator. The density functions of $H^{\alpha}(t)$ and $T_{2 \alpha}(t)$ coincides (see Meerschaert et al., 2011). Thus, we have

$$
\mathcal{N}_{c}^{\alpha}(t) \stackrel{d}{=} \mathcal{N}_{c}\left(H^{\alpha}(t)\right), \quad t>0
$$

where the inverse $\alpha$-stable subordinator is independent of the CPP.

Meerschaert et al. (2011) showed that the TFPP is a scaling limit of a continuous time random walk (CTRW). Here, we show that the CFPP occurs as a CTRW scaling limit.

Let $W_{1}, W_{2}, \ldots, W_{n}$ be iid waiting times such that $\operatorname{Pr}\left\{W_{n}>t\right\}=t^{-\alpha} L(t)$, where $0<\alpha<1$ and $L$ is a slowly varying function. For example, the Mittag-Leffler waiting times. There exist $b_{n}>0$ such that

$$
b_{n}\left(W_{1}+W_{2}+\cdots+W_{n}\right) \Rightarrow D^{\alpha}(1)
$$

where $\Rightarrow$ denotes convergence in distribution, that is, $W_{1}$ belongs to the strict domain of attraction of some stable law $D^{\alpha}(1)$. Let $b(t)=b_{[t]}$. Meerschaert et al. (2011) showed that there exists a regularly varying function $\tilde{b}$ with index $\alpha$ such that $1 / b(\tilde{b}(c)) \sim c$, as $c \rightarrow \infty$. Now, consider a renewal process

where $T_{n}=W_{1}+W_{2}+\cdots+W_{n}$.

$$
R(t)=\max \left\{n \geq 0: T_{n} \leq t\right\},
$$

Let $\left\{X_{i}\right\}_{i \geq 1}$ be a sequence of iid random variables whose distribution is given by (3.25) and $Y^{(p)}$ be a Bernoulli random variable independent of $\left\{X_{i}\right\}_{i \geq 1}$ such that $\operatorname{Pr}\left\{Y^{(p)}=1\right\}=p$ and 
$\operatorname{Pr}\left\{Y^{(p)}=0\right\}=1-p$. Let $Z_{i}=X_{i} Y^{(p)}, i \geq 1$. Note that $Z_{i}$ 's are iid random variables. Also, let $S^{(p)}(n)=\sum_{i=1}^{n} Z_{i}$. Then, $S^{(p)}(R(t))$ is a CTRW with heavy-tailed waiting times and jumps distributed according to the law of $Z_{1}$.

Theorem 4.8. The CFPP is the scaling limit of a CTRW. That is,

$$
\left\{S^{(1 / \tilde{b}(c))}\left(\left[\lambda_{0} R(c t)\right]\right)\right\}_{t \geq 0} \Rightarrow\left\{\mathcal{N}_{c}\left(H^{\alpha}(t)\right)\right\}_{t \geq 0}
$$

as $c \rightarrow \infty$ in the $M_{1}$ topology on $D([0, \infty), \mathbb{R})$.

Proof: On substituting $\alpha=1$ in (3.20), we get

$$
\mathbb{E}\left(e^{-w \mathcal{N}_{c}(t)}\right)=\exp \left(-t^{\alpha} \sum_{j=0}^{\infty}\left(\lambda_{j}-\lambda_{j-1}\right) e^{-w j}\right), \quad w \geq 0 .
$$

Also,

$$
\begin{aligned}
\mathbb{E}\left(e^{-w Z_{1}}\right)= & \sum_{j=0}^{\infty} e^{-w j} \operatorname{Pr}\left\{Z_{1}=j\right\} \\
& =\operatorname{Pr}\left\{X_{1} Y^{(p)}=0\right\}+\sum_{j=1}^{\infty} e^{-w j} \operatorname{Pr}\left\{X_{1} Y^{(p)}=j\right\} \\
& =\sum_{j=1}^{\infty} \operatorname{Pr}\left\{X_{1}=j, Y^{(p)}=0\right\}+\sum_{j=1}^{\infty} e^{-w j} \operatorname{Pr}\left\{X_{1}=j, Y^{(p)}=1\right\} \\
& =\sum_{j=1}^{\infty} \operatorname{Pr}\left\{X_{1}=j\right\} \operatorname{Pr}\left\{Y^{(p)}=0\right\}+\sum_{j=1}^{\infty} e^{-w j} \operatorname{Pr}\left\{X_{1}=j\right\} \operatorname{Pr}\left\{Y^{(p)}=1\right\} \\
& =1+\frac{p}{\lambda_{0}} \sum_{j=0}^{\infty}\left(\lambda_{j-1}-\lambda_{j}\right) e^{-w j}
\end{aligned}
$$

where the independence of $Y^{(p)}$ and $\left\{X_{i}\right\}_{i \geq 1}$ is used in the penultimate step. Thus,

$$
\begin{aligned}
\mathbb{E}\left(e^{-w S^{(p)}\left(\left[\lambda_{0} t / p\right]\right)}\right) & =\left(1+\frac{p}{\lambda_{0}} \sum_{j=0}^{\infty}\left(\lambda_{j-1}-\lambda_{j}\right) e^{-w j}\right)^{\left[\lambda_{0} t / p\right]} \\
& \rightarrow \exp \left(-t \sum_{j=0}^{\infty} e^{-w j}\left(\lambda_{j}-\lambda_{j-1}\right)\right)
\end{aligned}
$$

as $p \rightarrow 0$. Here, we have used the fact that $(1+a p)^{1 / p} \rightarrow e^{a}$ as $p \rightarrow 0$. Thus, $S^{(p)}\left(\left[\lambda_{0} t / p\right]\right) \Rightarrow \mathcal{N}_{c}(t)$ for any $t>0$.

From this stage the proof follows along the similar lines to that of Theorem 2.5 of Meerschaert et al. (2011) with the Lévy process $\left\{\mathcal{N}_{c}(t)\right\}_{t \geq 0}$ and the CTRW $\left\{S^{(1 / \tilde{b}(c))}\left(\left[\lambda_{0} R(c t)\right]\right)\right\}_{t \geq 0}$.

Next we show that the CPP can be obtained by time changing the CFPP by a suitable random time process whose one-dimensional distribution is given in terms of the $H$-function.

Cahoy and Polito (2012) defined a random time process $\left\{\mathcal{H}^{\alpha}(t)\right\}_{t>0}, 0<\alpha \leq 1$, whose density function $f_{\mathcal{H}^{\alpha}(t)}(x, t), x>0$, is given by

$$
f_{\mathcal{H}^{\alpha}(t)}(x, t)=t^{-\frac{1}{\alpha}} \mathrm{H}_{1,1}^{1,0}\left[t^{-\frac{1}{\alpha}} x \mid \begin{array}{c}
(1-\underset{1 / \alpha, 1 / \alpha)}{(0,1)}
\end{array}\right] .
$$


Also, it has the following Mellin transform:

$$
\int_{0}^{\infty} x^{\nu-1} f_{\mathcal{H}^{\alpha}(t)}(x, t) \mathrm{d} x=\frac{\Gamma(\nu) t^{\frac{\nu-1}{\alpha}}}{\Gamma(1-1 / \alpha+\nu / \alpha)}, \quad t>0, \nu \in \mathbb{R} .
$$

Here, $\mathrm{H}_{1,1}^{1,0}(\cdot)$ denotes the $H$-function, for more details on this function we refer the reader to Mathai et al. (2010).

Proposition 4.9. Let the process $\left\{\mathcal{H}^{\alpha}(t)\right\}_{t>0}, 0<\alpha \leq 1$, be independent of the CFPP $\left\{\mathcal{N}_{c}^{\alpha}(t)\right\}_{t>0}$. Then,

$$
\mathcal{N}_{c}(t) \stackrel{d}{=} \mathcal{N}_{c}^{\alpha}\left(\mathcal{H}^{\alpha}(t)\right), \quad t>0
$$

Proof: Using (3.8), we have

$$
\begin{aligned}
& \int_{0}^{\infty} G_{c}^{\alpha}(u, s) f_{\mathcal{H}^{\alpha}(t)}(s, t) \mathrm{d} s \\
& =\int_{0}^{\infty} \sum_{n=0}^{\infty} u^{n} p_{c}^{\alpha}(n, s) f_{\mathcal{H}^{\alpha}(t)}(s, t) \mathrm{d} s \\
& =\int_{0}^{\infty}\left(E_{\alpha, 1}\left(-\lambda_{0} s^{\alpha}\right)\right. \\
& \left.+\sum_{n=1}^{\infty} u^{n} \sum_{k=1}^{n} \sum_{\Lambda_{n}^{k}} k ! \prod_{j=1}^{n-k+1} \frac{\left(\lambda_{j-1}-\lambda_{j}\right)^{k_{j}}}{k_{j} !} s^{k \alpha} E_{\alpha, k \alpha+1}^{k+1}\left(-\lambda_{0} s^{\alpha}\right)\right) f_{\mathcal{H}^{\alpha}(t)}(s, t) \mathrm{d} s \\
& =\int_{0}^{\infty}\left(\frac{1}{2 \pi i} \int_{c-i \infty}^{c+i \infty} \frac{\Gamma(z) \Gamma(1-z)}{\Gamma(1-\alpha z)}\left(\lambda_{0} s^{\alpha}\right)^{-z} \mathrm{~d} z+\sum_{n=1}^{\infty} u^{n} \sum_{k=1}^{n} \sum_{\Lambda_{n}^{k}}^{n} \prod_{j=1}^{n-k+1} \frac{\left(\lambda_{j-1}-\lambda_{j}\right)^{k_{j}}}{k_{j} !}\right. \\
& \left.\cdot \frac{1}{2 \pi i} \int_{c-i \infty}^{c+i \infty} \frac{\Gamma(z) \Gamma(k+1-z)}{\Gamma(\alpha(k-z)+1)}\left(\lambda_{0} s^{\alpha}\right)^{-z} s^{k \alpha} \mathrm{d} z\right) f_{\mathcal{H}^{\alpha}(t)}(s, t) \mathrm{d} s, \quad \text { (using (2.7)) } \\
& =\frac{1}{2 \pi i} \int_{c-i \infty}^{c+i \infty} \frac{\Gamma(z) \Gamma(1-z) \lambda_{0}^{-z}}{\Gamma(1-\alpha z)}\left(\int_{0}^{\infty} s^{-\alpha z} f_{\mathcal{H}^{\alpha}(t)}(s, t) \mathrm{d} s\right) \mathrm{d} z \\
& +\sum_{n=1}^{\infty} u^{n} \sum_{k=1}^{n} \sum_{\Lambda_{n}^{k}}^{n-k+1} \prod_{j=1}^{n+1} \frac{\left(\lambda_{j-1}-\lambda_{j}\right)^{k_{j}}}{k_{j} !} \\
& \cdot \frac{1}{2 \pi i} \int_{c-i \infty}^{c+i \infty} \frac{\Gamma(z) \Gamma(k+1-z) \lambda_{0}^{-z}}{\Gamma(\alpha(k-z)+1)}\left(\int_{0}^{\infty} s^{k \alpha-\alpha z} f_{\mathcal{H}^{\alpha}(t)}(s, t) \mathrm{d} s\right) \mathrm{d} z \\
& =\frac{1}{2 \pi i} \int_{c-i \infty}^{c+i \infty} \Gamma(z)\left(\lambda_{0} t\right)^{-z} \mathrm{~d} z \\
& +\sum_{n=1}^{\infty} u^{n} \sum_{k=1}^{n} \sum_{\Lambda_{n}^{k}} \prod_{j=1}^{n-k+1} \frac{\left(\lambda_{j-1}-\lambda_{j}\right)^{k_{j}}}{k_{j} !} \frac{t^{k}}{2 \pi i} \int_{c-i \infty}^{c+i \infty} \Gamma(z)\left(\lambda_{0} t\right)^{-z} \mathrm{~d} z, \quad(\text { using }(4.5)) \\
& =e^{-t \lambda_{0}}+\sum_{n=1}^{\infty} u^{n} \sum_{k=1}^{n} \sum_{\Lambda_{n}^{k}} \prod_{j=1}^{n-k+1} \frac{\left(\lambda_{j-1}-\lambda_{j}\right)^{k_{j}}}{k_{j} !} t^{k} e^{-t \lambda_{0}}, \quad(\operatorname{using}(2.5)) \\
& =G_{c}(u, t) \text {, }
\end{aligned}
$$

where in the last step we have used (4.1).

\section{The dependence structure for CFPP and its increments}

In this section, we show that the CFPP has LRD property whereas its increments exhibits the SRD property.

For a non-stationary stochastic process $\{X(t)\}_{t \geq 0}$, the LRD and SRD properties are defined as follows (see D'Ovidio and Nane, 2014, Maheshwari and Vellaisamy, 2016): 
Definition 5.1. Let $s>0$ be fixed and $\{X(t)\}_{t \geq 0}$ be a stochastic process whose correlation function satisfies

$$
\operatorname{Corr}(X(s), X(t)) \sim c(s) t^{-\gamma}, \text { as } t \rightarrow \infty,
$$

for some $c(s)>0$. The process $\{X(t)\}_{t \geq 0}$ has the LRD property if $\gamma \in(0,1)$ and the SRD property if $\gamma \in(1,2)$.

First, we obtain the covariance of CFPP. Using Theorem 2.1 of Leonenko et al. (2014) and the subordination result (4.4), the covariance of CFPP can be obtained as follows:

$$
\begin{aligned}
\operatorname{Cov}\left(\mathcal{N}_{c}^{\alpha}(s), \mathcal{N}_{c}^{\alpha}(t)\right) & =\operatorname{Cov}\left(\mathcal{N}_{c}\left(H^{\alpha}(s)\right), \mathcal{N}_{c}\left(H^{\alpha}(t)\right)\right) \\
& =\operatorname{Var}\left(\mathcal{N}_{c}(1)\right) \mathbb{E}\left(H^{\alpha}(\min \{s, t\})\right)+\left(\mathbb{E}\left(\mathcal{N}_{c}(1)\right)\right)^{2} \operatorname{Cov}\left(H^{\alpha}(s), H^{\alpha}(t)\right),
\end{aligned}
$$

where we used Theorem 4.3 and the fact that the inverse $\alpha$-stable subordinator $\left\{H^{\alpha}(t)\right\}_{t \geq 0}$ is a non-decreasing process.

On using Theorem 2.1 of Leonenko et al. (2014), the mean and variance of CFPP can alternatively be obtained as follows:

and

$$
\mathbb{E}\left(\mathcal{N}_{c}^{\alpha}(t)\right)=\mathbb{E}\left(\mathcal{N}_{c}(1)\right) \mathbb{E}\left(H^{\alpha}(t)\right)
$$

$$
\operatorname{Var}\left(\mathcal{N}_{c}^{\alpha}(t)\right)=\left(\mathbb{E}\left(\mathcal{N}_{c}(1)\right)\right)^{2} \operatorname{Var}\left(H^{\alpha}(t)\right)+\operatorname{Var}\left(\mathcal{N}_{c}(1)\right) \mathbb{E}\left(H^{\alpha}(t)\right)
$$

where the mean and variance of inverse $\alpha$-stable subordinator are given by (see Leonenko et al., 2014, Eq. (8) and Eq. (11))

and

$$
\mathbb{E}\left(H^{\alpha}(t)\right)=\frac{t^{\alpha}}{\Gamma(\alpha+1)}
$$

$$
\operatorname{Var}\left(H^{\alpha}(t)\right)=t^{2 \alpha}\left(\frac{2}{\Gamma(2 \alpha+1)}-\frac{1}{\Gamma^{2}(\alpha+1)}\right)
$$

respectively.

Remark 5.2. From (3.13), (3.14) and (5.2), we get

$$
\operatorname{Var}\left(\mathcal{N}_{c}^{\alpha}(t)\right)-\mathbb{E}\left(\mathcal{N}_{c}^{\alpha}(t)\right)=\frac{2 t^{\alpha} \sum_{j=1}^{\infty} j \lambda_{j}}{\Gamma(\alpha+1)}+\left(\sum_{j=0}^{\infty} \lambda_{j}\right)^{2} \operatorname{Var}\left(H^{\alpha}(t)\right) .
$$

Thus, the CFPP exhibits overdispersion as $\operatorname{Var}\left(\mathcal{N}_{c}^{\alpha}(t)\right)-\mathbb{E}\left(\mathcal{N}_{c}^{\alpha}(t)\right)>0$ for $t>0$.

Let

$$
R=\frac{1}{\Gamma(\alpha+1)} \sum_{j=0}^{\infty} \lambda_{j}, \quad S=\left(\frac{2}{\Gamma(2 \alpha+1)}-\frac{1}{\Gamma^{2}(\alpha+1)}\right)\left(\sum_{j=0}^{\infty} \lambda_{j}\right)^{2}
$$

and

$$
T=\frac{1}{\Gamma(\alpha+1)}\left(\sum_{j=0}^{\infty} \lambda_{j}+2 \sum_{j=1}^{\infty} j \lambda_{j}\right)
$$

For $0<s \leq t$ in $(5.1)$, we get

$$
\operatorname{Cov}\left(\mathcal{N}_{c}^{\alpha}(s), \mathcal{N}_{c}^{\alpha}(t)\right)=T s^{\alpha}+\left(\sum_{j=0}^{\infty} \lambda_{j}\right)^{2} \operatorname{Cov}\left(H^{\alpha}(s), H^{\alpha}(t)\right) .
$$

For large $t$, the following result due to Leonenko et al. (2014):

$$
\operatorname{Cov}\left(H^{\alpha}(s), H^{\alpha}(t)\right) \sim \frac{s^{2 \alpha}}{\Gamma(2 \alpha+1)},
$$


is used to obtain

$$
\operatorname{Cov}\left(\mathcal{N}_{c}^{\alpha}(s), \mathcal{N}_{c}^{\alpha}(t)\right) \sim T s^{\alpha}+\frac{\left(\sum_{j=0}^{\infty} \lambda_{j}\right)^{2} s^{2 \alpha}}{\Gamma(2 \alpha+1)} \text { as } t \rightarrow \infty .
$$

We now show that the CFPP has LRD property.

Theorem 5.3. The CFPP exhibits the LRD property.

Proof: Using (3.14) and (5.4), we get the following for fixed $s>0$ and large $t$ :

$$
\begin{aligned}
\operatorname{Corr}\left(\mathcal{N}_{c}^{\alpha}(s), \mathcal{N}_{c}^{\alpha}(t)\right) & =\frac{\operatorname{Cov}\left(\mathcal{N}_{c}^{\alpha}(s), \mathcal{N}_{c}^{\alpha}(t)\right)}{\sqrt{\operatorname{Var}\left(\mathcal{N}_{c}^{\alpha}(s)\right)} \sqrt{\operatorname{Var}\left(\mathcal{N}_{c}^{\alpha}(t)\right)}} \\
& \sim \frac{\Gamma(2 \alpha+1) T s^{\alpha}+\left(\sum_{j=0}^{\infty} \lambda_{j}\right)^{2} s^{2 \alpha}}{\Gamma(2 \alpha+1) \sqrt{\operatorname{Var}\left(\mathcal{N}_{c}^{\alpha}(s)\right)} \sqrt{S t^{2 \alpha}+T t^{\alpha}}} \\
& \sim c_{0}(s) t^{-\alpha},
\end{aligned}
$$

where

$$
c_{0}(s)=\frac{\Gamma(2 \alpha+1) T s^{\alpha}+\left(\sum_{j=0}^{\infty} \lambda_{j}\right)^{2} s^{2 \alpha}}{\Gamma(2 \alpha+1) \sqrt{\operatorname{Var}\left(\mathcal{N}_{c}^{\alpha}(s)\right)} \sqrt{S}} .
$$

As $0<\alpha<1$, the result follows.

Remark 5.4. For $0<\alpha<1$, we have shown that the CFPP has the LRD property. Similarly, it can be shown that the CPP exhibits the LRD property.

For a fixed $\delta>0$, we define the convoluted fractional Poissonian noise (CFPN), denoted by $\left\{Z_{c, \delta}^{\alpha}(t)\right\}_{t \geq 0}$, as the increment process of CFPP, that is,

$$
Z_{c, \delta}^{\alpha}(t):=\mathcal{N}_{c}^{\alpha}(t+\delta)-\mathcal{N}_{c}^{\alpha}(t)
$$

Next, we show that the CFPN exhibits the SRD property.

Theorem 5.5. The CFPN has the SRD property.

Proof: Let $s>0$ be fixed such that $0<s+\delta \leq t$. We have,

$$
\begin{aligned}
\operatorname{Cov}\left(Z_{c, \delta}^{\alpha}(s), Z_{c, \delta}^{\alpha}(t)\right)= & \operatorname{Cov}\left(\mathcal{N}_{c}^{\alpha}(s+\delta)-\mathcal{N}_{c}^{\alpha}(s), \mathcal{N}_{c}^{\alpha}(t+\delta)-\mathcal{N}_{c}^{\alpha}(t)\right) \\
= & \operatorname{Cov}\left(\mathcal{N}_{c}^{\alpha}(s+\delta), \mathcal{N}_{c}^{\alpha}(t+\delta)\right)+\operatorname{Cov}\left(\mathcal{N}_{c}^{\alpha}(s), \mathcal{N}_{c}^{\alpha}(t)\right) \\
& -\operatorname{Cov}\left(\mathcal{N}_{c}^{\alpha}(s+\delta), \mathcal{N}_{c}^{\alpha}(t)\right)-\operatorname{Cov}\left(\mathcal{N}_{c}^{\alpha}(s), \mathcal{N}_{c}^{\alpha}(t+\delta)\right)
\end{aligned}
$$

Leonenko et al. (2014) obtained the following expression for the covariance of inverse $\alpha$-stable subordinator:

$$
\operatorname{Cov}\left(H^{\alpha}(s), H^{\alpha}(t)\right)=\frac{1}{\Gamma^{2}(\alpha+1)}\left(\alpha s^{2 \alpha} B(\alpha, \alpha+1)+F(\alpha ; s, t)\right),
$$

where $F(\alpha ; s, t)=\alpha t^{2 \alpha} B(\alpha, \alpha+1 ; s / t)-(t s)^{\alpha}$. Here, $B(\alpha, \alpha+1)$ is the beta function whereas $B(\alpha, \alpha+1 ; s / t)$ is the incomplete beta function.

On substituting (5.6) in (5.3) and using the following asymptotic result (see Maheshwari and Vellaisamy, 2016, Eq. (8)):

we obtain

$$
F(\alpha ; s, t) \sim \frac{-\alpha^{2}}{(\alpha+1)} \frac{s^{\alpha+1}}{t^{1-\alpha}}, \text { as } t \rightarrow \infty
$$

$$
\operatorname{Cov}\left(\mathcal{N}_{c}^{\alpha}(s), \mathcal{N}_{c}^{\alpha}(t)\right) \sim T s^{\alpha}+R^{2}\left(\alpha s^{2 \alpha} B(\alpha, \alpha+1)-\frac{\alpha^{2}}{(\alpha+1)} \frac{s^{\alpha+1}}{t^{1-\alpha}}\right) \quad \text { as } t \rightarrow \infty
$$


From (5.5) and (5.7), we get the following for large $t$ :

$$
\begin{aligned}
\operatorname{Cov}\left(Z_{c, \delta}^{\alpha}(s), Z_{c, \delta}^{\alpha}(t)\right) & \sim \frac{R^{2} \alpha^{2}}{\alpha+1}\left(\frac{s^{\alpha+1}}{(t+\delta)^{1-\alpha}}+\frac{(s+\delta)^{\alpha+1}}{t^{1-\alpha}}-\frac{s^{\alpha+1}}{t^{1-\alpha}}-\frac{(s+\delta)^{\alpha+1}}{(t+\delta)^{1-\alpha}}\right) \\
& =\frac{R^{2} \alpha^{2}}{\alpha+1}\left((t+\delta)^{\alpha-1}-t^{\alpha-1}\right)\left(s^{\alpha+1}-(s+\delta)^{\alpha+1}\right) \\
& \sim \frac{\alpha^{2} \delta(1-\alpha)}{\alpha+1}\left((s+\delta)^{\alpha+1}-s^{\alpha+1}\right) R^{2} t^{\alpha-2} .
\end{aligned}
$$

Now,

$$
\operatorname{Var}\left(Z_{c, \delta}^{\alpha}(t)\right)=\operatorname{Var}\left(\mathcal{N}_{c}^{\alpha}(t+\delta)\right)+\operatorname{Var}\left(\mathcal{N}_{c}^{\alpha}(t)\right)-2 \operatorname{Cov}\left(\mathcal{N}_{c}^{\alpha}(t), \mathcal{N}_{c}^{\alpha}(t+\delta)\right) .
$$

From (5.3) and (5.6), we have

$$
\operatorname{Cov}\left(\mathcal{N}_{c}^{\alpha}(t), \mathcal{N}_{c}^{\alpha}(t+\delta)\right)=T t^{\alpha}+R^{2}\left(\alpha t^{2 \alpha} B(\alpha, \alpha+1)+F(\alpha ; t, t+\delta)\right),
$$

where $F(\alpha ; t, t+\delta)=\alpha(t+\delta)^{2 \alpha} B(\alpha, \alpha+1 ; t /(t+\delta))-(t(t+\delta))^{\alpha}$.

For large $t$, we have

$$
B(\alpha, \alpha+1 ; t /(t+\delta)) \sim B(\alpha, \alpha+1)=\frac{\Gamma(\alpha) \Gamma(\alpha+1)}{\Gamma(2 \alpha+1)}
$$

Substituting (3.14) and (5.10) in (5.9), we get

$$
\begin{aligned}
\operatorname{Var}\left(Z_{c, \delta}^{\alpha}(t)\right) & \sim\left(S-2 R^{2} \alpha B(\alpha, \alpha+1)\right) t^{2 \alpha}+\left(S-2 R^{2} \alpha B(\alpha, \alpha+1)\right)(t+\delta)^{2 \alpha} \\
& +T\left((t+\delta)^{\alpha}-t^{\alpha}\right)+2 R^{2}(t(t+\delta))^{\alpha} \\
& =T t^{\alpha}\left(\left(1+\frac{\delta}{t}\right)^{\alpha}-1\right)-R^{2} t^{2 \alpha}\left(\left(1+\frac{\delta}{t}\right)^{\alpha}-1\right)^{2} \\
& \sim T \alpha \delta t^{\alpha-1}-R^{2} \alpha^{2} \delta^{2} t^{2 \alpha-2} \\
& \sim \alpha \delta T t^{\alpha-1}, \text { as } t \rightarrow \infty .
\end{aligned}
$$

From (5.8) and (5.11), we have

$$
\begin{aligned}
\operatorname{Corr}\left(Z_{c, \delta}^{\alpha}(s), Z_{c, \delta}^{\alpha}(t)\right) & \sim \frac{\alpha^{2} \delta(1-\alpha)\left((s+\delta)^{\alpha+1}-s^{\alpha+1}\right) R^{2} t^{\alpha-2}}{(\alpha+1) \sqrt{\operatorname{Var}\left(Z_{c, \delta}^{\alpha}(s)\right)} \sqrt{\alpha \delta T t^{\alpha-1}}} \\
& =c_{1}(s) t^{-(3-\alpha) / 2}, \text { as } t \rightarrow \infty
\end{aligned}
$$

where

$$
c_{1}(s)=\frac{\alpha^{2} \delta(1-\alpha)\left((s+\delta)^{\alpha+1}-s^{\alpha+1}\right) R^{2}}{(\alpha+1) \sqrt{\operatorname{Var}\left(Z_{c, \delta}^{\alpha}(s)\right)} \sqrt{\alpha \delta T}} .
$$

Thus, the CFPN exhibits the SRD property as $1<(3-\alpha) / 2<3 / 2$.

\section{Acknowledgment}

We thank the anonymous referees for their insightful comments and suggestions that have led to improvements. 


\section{References}

Aletti, G., Leonenko, N., and Merzbach, E. Fractional Poisson fields and martingales. J. Stat. Phys., 170 (4), 700-730 (2018). MR3764004.

Beghin, L. Random-time processes governed by differential equations of fractional distributed order. Chaos Solitons Fractals, 45 (11), 1314-1327 (2012). MR2990245.

Beghin, L. and Macci, C. Fractional discrete processes: compound and mixed Poisson representations. J. Appl. Probab., 51 (1), 19-36 (2014). MR3189439.

Beghin, L. and Orsingher, E. Fractional Poisson processes and related planar random motions. Electron. J. Probab., 14, paper no. 61, 1790-1827 (2009). MR2535014.

Beghin, L. and Orsingher, E. Poisson-type processes governed by fractional and higher-order recursive differential equations. Electron. J. Probab., 15, paper no. 22, 684-709 (2010). MR2650778.

Beghin, L. and Vellaisamy, P. Space-fractional versions of the negative binomial and Polya-type processes. Methodol. Comput. Appl. Probab., 20 (2), 463-485 (2018). MR3803930.

Biard, R. and Saussereau, B. Fractional Poisson process: long-range dependence and applications in ruin theory. J. Appl. Probab., 51 (3), 727-740 (2014). MR3256223.

Cahoy, D. O. and Polito, F. Simulation and estimation for the fractional Yule process. Methodol. Comput. Appl. Probab., 14 (2), 383-403 (2012). MR2912344.

Comtet, L. Advanced combinatorics. The art of finite and infinite expansions. D. Reidel Publishing Co., Dordrecht (1974). ISBN 90-277-0441-4. MR0460128.

Damelin, S. B. and Miller, W., Jr. The mathematics of signal processing. Cambridge Texts in Applied Mathematics. Cambridge University Press, Cambridge (2012). ISBN 978-1-107-60104-8. MR2883645.

Di Crescenzo, A., Martinucci, B., and Meoli, A. A fractional counting process and its connection with the Poisson process. ALEA Lat. Am. J. Probab. Math. Stat., 13 (1), 291-307 (2016). MR3479478.

D'Ovidio, M. and Nane, E. Time dependent random fields on spherical non-homogeneous surfaces. Stochastic Process. Appl., 124 (6), 2098-2131 (2014). MR3188350.

Garra, R., Orsingher, E., and Polito, F. State-dependent fractional point processes. J. Appl. Probab., 52 (1), 18-36 (2015). MR3336844.

Johnson, W. P. The curious history of Faà di Bruno's formula. Amer. Math. Monthly, 109 (3), 217-234 (2002). MR1903577.

Kataria, K. K. and Khandakar, M. On the long-range dependence of mixed fractional Poisson process. J. Theoret. Probab., (2020). DOI: 10.1007/s10959-020-01015-y.

Kataria, K. K. and Vellaisamy, P. Correlation between Adomian and partial exponential Bell polynomials. C. R. Math. Acad. Sci. Paris, 355 (9), 929-936 (2017a). MR3709529.

Kataria, K. K. and Vellaisamy, P. Saigo space-time fractional Poisson process via Adomian decomposition method. Statist. Probab. Lett., 129, 69-80 (2017b). MR3688517.

Kilbas, A. A., Srivastava, H. M., and Trujillo, J. J. Theory and applications of fractional differential equations, volume 204 of North-Holland Mathematics Studies. Elsevier Science B.V., Amsterdam (2006). ISBN 978-0-444-51832-3; 0-444-51832-0. MR2218073.

Laskin, N. Fractional Poisson process. Chaotic transport and complexity in classical and quantum dynamics. Commun. Nonlinear Sci. Numer. Simul., 8 (3-4), 201-213 (2003). MR2007003.

Leonenko, N. N., Meerschaert, M. M., Schilling, R. L., and Sikorskii, A. Correlation structure of time-changed Lévy processes. Commun. Appl. Ind. Math., 6 (1), e-483, 22 (2014). MR3277310.

Maheshwari, A. and Vellaisamy, P. On the long-range dependence of fractional Poisson and negative binomial processes. J. Appl. Probab., 53 (4), 989-1000 (2016). MR3581236.

Mathai, A. M. and Haubold, H. J. Special functions for applied scientists. Springer, New York (2008). DOI: 10.1007/978-0-387-75894-7. 
Mathai, A. M., Saxena, R. K., and Haubold, H. J. The H-function. Theory and applications. Springer, New York (2010). ISBN 978-1-4419-0915-2. MR2562766.

Meerschaert, M. M., Nane, E., and Vellaisamy, P. The fractional Poisson process and the inverse stable subordinator. Electron. J. Probab., 16, paper no. 59, 1600-1620 (2011). MR2835248.

Orsingher, E. and Beghin, L. Time-fractional telegraph equations and telegraph processes with Brownian time. Probab. Theory Related Fields, 128 (1), 141-160 (2004). MR2027298.

Orsingher, E. and Polito, F. Fractional pure birth processes. Bernoulli, 16 (3), 858-881 (2010). MR2730651.

Orsingher, E. and Polito, F. The space-fractional Poisson process. Statist. Probab. Lett., 82 (4), 852-858 (2012). MR2899530.

Orsingher, E. and Toaldo, B. Counting processes with Bernštein intertimes and random jumps. $J$. Appl. Probab., 52 (4), 1028-1044 (2015). MR3439170.

Paris, R. B. and Kaminski, D. Asymptotics and Mellin-Barnes integrals, volume 85 of Encyclopedia of Mathematics and its Applications. Cambridge University Press, Cambridge (2001). ISBN 0-521-79001-8. MR1854469.

Polito, F. and Scalas, E. A generalization of the space-fractional Poisson process and its connection to some Lévy processes. Electron. Commun. Probab., 21, paper no. 20, pp. 14 (2016). MR3485389. 